ja074728s_SI_0824.pdf

Supporting Information for:

\title{
A Silicon-Based Approach to Oligoarenes by Iterative Cross-Coupling Reactions of Halogenated Organo[(2-hydroxymethyl)phenyl]dimethylsilanes
}

\author{
Yoshiaki Nakao,* Jinshui Chen, Masaaki Tanaka, and Tamejiro Hiyama* \\ Department of Material Chemistry, Graduate School of Engineering, \\ Kyoto University, Kyoto 615-8510 Japan
}

General. All manipulations of oxygen- and moisture-sensitive materials were conducted with a standard Schlenk technique or in a dry box under an argon atmosphere. Flush column chromatography was performed using Kanto Chemical silica gel 60 (spherical, 40-50 $\mu \mathrm{m}$ ). Analytical thin layer chromatography (TLC) was performed on Merck Kieselgel $60 \mathrm{~F}_{254}(0.25 \mathrm{~mm})$ plates. Visualization was accomplished with UV light $(254 \mathrm{~nm})$ and/or an aqueous alkaline $\mathrm{KMnO}_{4}$ solution followed by heating.

Apparatus. Proton and carbon nuclear magnetic resonance spectra $\left({ }^{1} \mathrm{H}\right.$ NMR and ${ }^{13} \mathrm{C}$ NMR $)$ were recorded on a Varian Mercury $400\left({ }^{1} \mathrm{H}, 400 \mathrm{MHz} ;{ }^{13} \mathrm{C}, 101 \mathrm{MHz}\right)$ spectrometer with solvent resonance $\left({ }^{1} \mathrm{H} \mathrm{NMR}, \mathrm{CHCl}_{3}\right.$ at $7.26 \mathrm{ppm} ;{ }^{13} \mathrm{C} \mathrm{NMR}, \mathrm{CDCl}_{3}$ at $\left.77.0 \mathrm{ppm}\right)$ as the internal standard. ${ }^{1} \mathrm{H}$ NMR data are reported as follows: chemical shift, multiplicity $(\mathrm{s}=$ singlet, $\mathrm{d}=$ doublet, $\mathrm{t}=$ triplet, $\mathrm{q}=$ quartet, quint $=$ quintet, sext $=$ sextet, $\mathrm{m}=$ multiplet, $\mathrm{br}=$ broad $)$, coupling constants $(\mathrm{Hz})$, and integration. IR spectra recorded on a Shimadzu FT-IR 8400 spectrometer are reported in $\mathrm{cm}^{-1}$. Melting points (mp) were measured on a Yanaco Mp-500D and are uncorrected. Elemental analyses were performed by Elemental Analysis Center of Kyoto University. High-resolution mass spectra were obtained with a JEOL JMS-700 (EI) or JEOL JMS-HX110A (FAB+) spectrometer. Preparative recycling gel permeation chromatography (GPC) was performed with a JAI LC-908 chromatograph equipped with JAIGEL-1H and $-2 \mathrm{H}$ using chloroform as an eluent.

Chemicals. Unless otherwise noted, reagents were commercially available and were used without purification. Anhydrous DMF was purchased from Nacalai Tesque. Anhydrous toluene purchased from Kanto Chemical was degassed by purging vigorously with argon for 20 min and further purified by passage through activated alumina under positive argon pressure as described by Grubbs et al. ${ }^{1}$ RuPhos was prepared according to the Buchwald's protocol. ${ }^{2}$ Unless otherwise described below, preparation of organo[2-(hydroxymethyl)phenyl]dimethylsilanes is described in our previous publications. $^{3}$

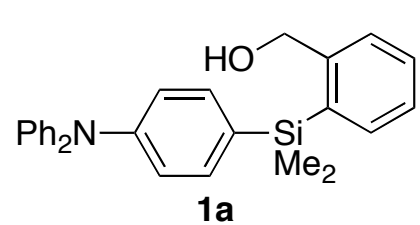

Preparation of [4-(diphenylamino)phenyl][2-(hydroxymethyl)phenyl]dimethylsilane (1a). ${ }^{4}$ To a suspension of $\mathrm{Mg}(1.9 \mathrm{~g}, 79 \mathrm{mmol})$ in $\mathrm{Et}_{2} \mathrm{O}$ (160 $\mathrm{mL})$ was added a solution of 4-bromo- $N, N$-diphenylaniline $(25 \mathrm{~g}, 77 \mathrm{mmol})$ in THF $(60 \mathrm{~mL})$ at $\mathrm{rt}$, and the resulting mixture was stirred at $60{ }^{\circ} \mathrm{C}$ for $4 \mathrm{~h}$. The mixture was cooled to $0{ }^{\circ} \mathrm{C}$, and then 1,1-dimethyl-2-oxa-1-silaindan 4 (12.1 g, $73 \mathrm{mmol}$ ) was added. After being stirred at $\mathrm{rt}$ overnight, the reaction mixture was quenched with a saturated $\mathrm{NH}_{4} \mathrm{Cl}$ aqueous solution at $0{ }^{\circ} \mathrm{C}$. The aqueous layer was extracted with diethyl ether, and the combined organic layers were dried over anhydrous $\mathrm{MgSO}_{4}$, concentrated in vacuo. The residue was purified by flash chromatography on silica gel to afford 1a (30 g, 95\%) as a colorless viscous oil, $\mathrm{R}_{\mathrm{f}} 0.70$ (hexane-ethyl acetate $\left.=1: 1\right)$. ${ }^{1} \mathrm{H}$ NMR $\left(400 \mathrm{MHz}, \mathrm{CDCl}_{3}\right) \delta 7.58(\mathrm{~d}, J=7.3 \mathrm{~Hz}$, $1 \mathrm{H}), 7.48-7.37(\mathrm{~m}, 2 \mathrm{H}), 7.31(\mathrm{~d}, J=8.4 \mathrm{~Hz}, 2 \mathrm{H}), 7.23(\mathrm{t}, J=7.8 \mathrm{~Hz}, 5 \mathrm{H}), 7.08(\mathrm{~d}, J=8.2 \mathrm{~Hz}, 4 \mathrm{H}), 7.01$ 
$(\mathrm{t}, J=7.5 \mathrm{~Hz}, 4 \mathrm{H}), 4.58(\mathrm{~s}, 2 \mathrm{H}), 1.33(\mathrm{br}, 1 \mathrm{H}), 0.59(\mathrm{~s}, 6 \mathrm{H}) ;{ }^{13} \mathrm{C} \mathrm{NMR}\left(101 \mathrm{MHz}, \mathrm{CDCl}_{3}\right) \delta 148.6$, 147.2 , 146.3, 136.2, 135.3, 134.6, 130.9, 129.8, 129.2, 128.2, 126.9, 124.7, 123.1, 122.1, 65.4, -0.8 ; IR (neat): 3398, 3057, 3011, 2955, 1585, 1489, 1327, 1315, 1279, 1254, 1196, 1109, 1076, 1028, 812, 754, 696, 665, $621 \mathrm{~cm}^{-1}$; Anal. Calcd for $\mathrm{C}_{27} \mathrm{H}_{27} \mathrm{NOSi}$; C, 79.17; H, 6.64. Found: C, 79.21; H, 6.80.

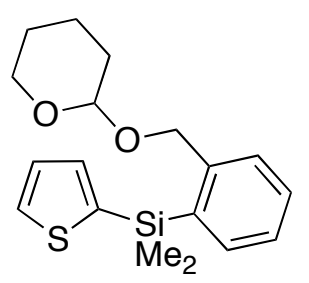

Preparation of dimethyl[2-(tetrahydro-2H-pyranoxymethyl)phenyl](2-thienyl)silane. To a mixture of $\mathbf{1 b}(20 \mathrm{~g}, 80 \mathrm{mmol})$ and 3,4-dihydro- $2 H$-pyran $(13.5 \mathrm{~g}, 160$ $\mathrm{mmol}$ ) were added 4 drops of conc. $\mathrm{HCl}$ at $\mathrm{rt}$, and the resulting mixture was stirred for $4 \mathrm{~h}$, diluted with $\mathrm{Et}_{2} \mathrm{O}$, neutralized with $\mathrm{NaHCO}_{3}$, dried over anhydrous $\mathrm{MgSO}_{4}$, and filtered through a Celite pad. After concentration in vacuo, the residue was purified by flash chromatography on silica gel to afford the title compound (22 $\mathrm{g}$, $82 \%)$ as a colorless oil, $\mathrm{R}_{\mathrm{f}} 0.30$ (hexane-ethyl acetate $\left.=20: 1\right) .{ }^{1} \mathrm{H}$ NMR $\left(400 \mathrm{MHz}, \mathrm{CDCl}_{3}\right) \delta 7.60$ (dd, $J=4.6,0.6 \mathrm{~Hz}, 1 \mathrm{H}), 7.53-7.46(\mathrm{~m}, 2 \mathrm{H}), 7.40(\mathrm{td}, J=7.5,1.3 \mathrm{~Hz}, 1 \mathrm{H}), 7.29-7.22(\mathrm{~m}, 2 \mathrm{H}), 7.17(\mathrm{dd}, J=$ $4.5,3.4 \mathrm{~Hz}, 1 \mathrm{H}), 4.73(\mathrm{~d}, J=12.1 \mathrm{~Hz}, 1 \mathrm{H}), 4.53(\mathrm{t}, J=3.5 \mathrm{~Hz}, 1 \mathrm{H}), 4.47(\mathrm{~d}, J=12.1 \mathrm{~Hz}, 1 \mathrm{H})$, 3.85-3.77 (m, 1H), 3.50-3.42 (m, 1H), 1.88-1.75 (m, 1H), 1.72-1.42 (m, 5H), 0.652 (s, 3H), $0.651(\mathrm{~s}$, $3 \mathrm{H}) ;{ }^{13} \mathrm{C}$ NMR $\left(101 \mathrm{MHz}, \mathrm{CDCl}_{3}\right) \delta 143.9,138.2,135.7,135.20,135.15,130.9,129.7,128.5,128.0$, 126.7, 97.8, 68.7, 62.1, 30.6, 25.6, 19.5, 0.4, 0.3; IR (neat): 2943, 2870, 1437, 1406, 1350, 1252, 1213, 1202, 1119, 1078, 1026, 989, 907, 833, 812, 777, 756, 708, $656 \mathrm{~cm}^{-1}$; Anal. Calcd for $\mathrm{C}_{18} \mathrm{H}_{24} \mathrm{O}_{2} \mathrm{SSi}$ : C, 65.01; H, 7.27. Found: C, 64.88; H, 7.06.

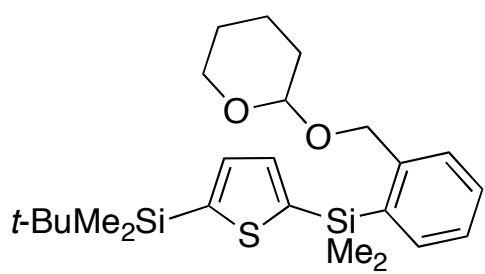

Preparation of 5-(tert-butyldimethylsilyl)-2-(dimethyl[2-(tetrahydro2H-pyranoxymethyl)phenyl]silyl)thiophene. To a solution of dimethyl[2-(tetrahydro-2H-pyranoxymethyl)phenyl](2-thienyl)silane $(1.56 \mathrm{~g}, 4.7 \mathrm{mmol})$ in $\mathrm{Et}_{2} \mathrm{O}(5 \mathrm{~mL})$ were added TMEDA $(0.63 \mathrm{~g}, 5.4$ $\mathrm{mmol})$ and a $1.6 \mathrm{M}$ solution of $n$-BuLi $(5.2 \mathrm{mmol})$ in hexane at $-40{ }^{\circ} \mathrm{C}$. The resulting mixture was stirred at $\mathrm{rt}$ for $1 \mathrm{~h}$, and then a solution of $t$ - $\mathrm{BuMe}_{2} \mathrm{SiCl}$ in $\mathrm{Et}_{2} \mathrm{O}(4 \mathrm{~mL})$ was added at $-40{ }^{\circ} \mathrm{C}$. The resulting mixture was stirred at the same temperature for $30 \mathrm{~min}$ and then at $\mathrm{rt}$ overnight, diluted with diethyl ether, washed with water and brine, and then dried over anhydrous $\mathrm{MgSO}_{4}$. Concentration in vacuo followed by flash chromatography on silica gel afforded the title compound $(1.74 \mathrm{~g}, 83 \%)$ as a colorless oil, $\mathrm{R}_{\mathrm{f}}$ 0.37 (hexane-ethyl acetate $=5: 1) .{ }^{1} \mathrm{H}$ NMR $\left(400 \mathrm{MHz}, \mathrm{CDCl}_{3}\right) \delta 7.56-7.43(\mathrm{~m}, 2 \mathrm{H}), 7.39(\mathrm{td}, J=7.5$, $1.4 \mathrm{~Hz}, 1 \mathrm{H}), 7.32-7.18(\mathrm{~m}, 3 \mathrm{H}), 4.72(\mathrm{~d}, J=12.1 \mathrm{~Hz}, 1 \mathrm{H}), 4.52(\mathrm{t}, J=3.6 \mathrm{~Hz}, 1 \mathrm{H}), 4.46(\mathrm{~d}, J=12.1 \mathrm{~Hz}$, $1 \mathrm{H}), 3.86-3.75(\mathrm{~m}, 1 \mathrm{H}), 3.50-3.39(\mathrm{~m}, 1 \mathrm{H}), 1.87-1.74(\mathrm{~m}, 1 \mathrm{H}), 1.70-1.40(\mathrm{~m}, 5 \mathrm{H}), 0.91(\mathrm{~s}, 9 \mathrm{H}), 0.653$ $(\mathrm{s}, 3 \mathrm{H}), 0.649(\mathrm{~s}, 3 \mathrm{H}), 0.29(\mathrm{~s}, 6 \mathrm{H}) ;{ }^{13} \mathrm{C} \mathrm{NMR}\left(101 \mathrm{MHz}, \mathrm{CDCl}_{3}\right) \delta 143.91,143.85,143.4,136.04$, 135.98, 135.9, 135.1, 129.6, 128.4, 126.7, 97.8, 68.8, 62.1, 30.6, 26.5, 25.6, 19.5, 17.0, 0.6, 0.4, -4.5; IR (neat): 2953, 2928, 2855, 1470, 1250, 1202, 1119, 1078, 1055, 1026, 1007, 976, 907, 835, 804, 773, 752, $675 \mathrm{~cm}^{-1}$; Anal. Calcd for $\mathrm{C}_{24} \mathrm{H}_{38} \mathrm{O}_{2} \mathrm{SSi}_{2} ; \mathrm{C}, 64.52 ; \mathrm{H}, 8.57$. Found: $\mathrm{C}, 64.51 ; \mathrm{H}, 8.37$.

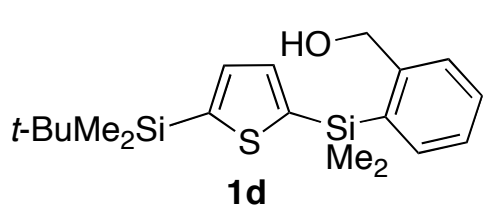

Preparation of 5-(tert-butyldimethylsilyl)-2-([2-(hydroxymethyl)phenyl]dimethylsilyl)thiophene (1d). A solution of 5-(tert-Butyldimethylsilyl)-2-(dimethyl[2-(tetrahydro-2H-pyranoxymeth yl)phenyl]silyl)thiophene (1.51 g, $34 \mathrm{mmol})$ and pyridinium p-toluenesulfonate (PPTS, $171 \mathrm{mg}, 0.68 \mathrm{mmol})$ in methanol $(10 \mathrm{~mL})$ was stirred at $40{ }^{\circ} \mathrm{C}$ for $2 \mathrm{~h}$ and then concentrated in vacuo. The residue was purified by flash chromatography on silica gel to afford $\mathbf{1 d}(1.13 \mathrm{~g}, 92 \%)$ as a white solid, $\mathrm{mp} 50.3-51.5^{\circ} \mathrm{C}, \mathrm{R}_{\mathrm{f}} 0.26$ (hexane-ethyl acetate = 5:1). ${ }^{1} \mathrm{H} \mathrm{NMR}\left(400 \mathrm{MHz}, \mathrm{CDCl}_{3}\right) \delta 7.56(\mathrm{~d}, J=7.3 \mathrm{~Hz}, 1 \mathrm{H}), 7.47-7.38(\mathrm{~m}$, 2H), 7.36-7.25 (m, 3H), $4.61(\mathrm{~d}, J=6.2 \mathrm{~Hz}, 2 \mathrm{H}), 1.38(\mathrm{t}, J=6.0 \mathrm{~Hz}, 1 \mathrm{H}), 0.90(\mathrm{~s}, 9 \mathrm{H}), 0.67(\mathrm{~s}, 6 \mathrm{H})$, $0.29(\mathrm{~s}, 6 \mathrm{H}) ;{ }^{13} \mathrm{C}$ NMR $\left(101 \mathrm{MHz}, \mathrm{CDCl}_{3}\right) \delta 146.4,144.0,143.9,136.2,136.0,135.7,135.1,130.0$, 
ja074728s_SI_0824.pdf

128.3, 127.0, 65.4, 26.5, 17.0, 0.5, -4.6; IR (KBr): 3321, 2953, 2928, 2856, 1250, 1204, 1007, 835, 822, $808,777 \mathrm{~cm}^{-1}$; Anal. Calcd for $\mathrm{C}_{19} \mathrm{H}_{30} \mathrm{OSSi}_{2}$; C, 62.92; H, 8.34. Found: C, 62.89; H, 8.17.

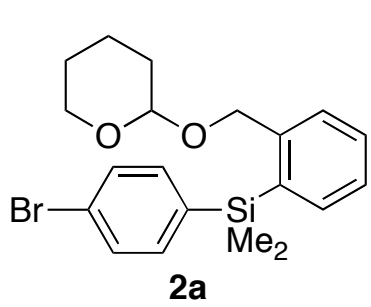

\section{Preparation of (4-bromophenyl)dimethyl[2-(tetrahydro-2H-pyranoxy-} methyl)phenyl]silane (2a). To a mixture of (4-bromophenyl)[2-(hydroxymethyl)phenyl]dimethylsilane (6.4 g, $20 \mathrm{mmol})$ and 3,4-dihydro- $2 \mathrm{H}$-pyran $(2.0 \mathrm{~g}, 24 \mathrm{mmol})$ was added a drop of conc. $\mathrm{HCl}$ at $\mathrm{rt}$, and the resulting mixture was stirred overnight. The mixture was diluted with $\mathrm{Et}_{2} \mathrm{O}$, neutralized with $\mathrm{NaHCO}_{3}$, dried over anhydrous $\mathrm{MgSO}_{4}$, and filtered through a Celite pad. After concentration in vacuo, the residue was purified by flash chromatography on silica gel to afford $\mathbf{2 a}(7.3 \mathrm{~g}, 90 \%)$ as a colorless oil, $\mathrm{R}_{\mathrm{f}} 0.29$ (hexane-ethyl acetate $=10: 1) .{ }^{1} \mathrm{H}$ NMR $\left(400 \mathrm{MHz}, \mathrm{CDCl}_{3}\right) \delta 7.54-7.37(\mathrm{~m}, 5 \mathrm{H}), 7.36-7.25(\mathrm{~m}, 3 \mathrm{H})$, $4.62(\mathrm{~d}, J=12.1 \mathrm{~Hz}, 1 \mathrm{H}), 4.43(\mathrm{t}, J=3.3 \mathrm{~Hz}, 1 \mathrm{H}), 4.33(\mathrm{~d}, J=11.9 \mathrm{~Hz}, 1 \mathrm{H}), 3.78-3.68(\mathrm{~m}, 1 \mathrm{H})$, 3.45-3.36 (m, 1H), 1.84-1.70 (m, 1H), 1.67-1.40 (m, 5H), $0.58(\mathrm{~s}, 3 \mathrm{H}), 0.57(\mathrm{~s}, 3 \mathrm{H}) ;{ }^{13} \mathrm{C}$ NMR $(101$ $\left.\mathrm{MHz}, \mathrm{CDCl}_{3}\right) \delta 143.9,137.7,135.5,135.3,130.8,129.7,128.6,126.8,123.7,97.8,68.7,62.1,30.6$, 25.5, 19.5, -0.9, -1.0; IR (neat): 2943, 2870, 1570, 1479, 1439, 1377, 1258, 1202, 1119, 1078, 1067, 1026, 1011, 974, 907, 835, 818, 806, 775, 754, $723 \mathrm{~cm}^{-1}$; Anal. Calcd for $\mathrm{C}_{20} \mathrm{H}_{25} \mathrm{BrO}_{2} \mathrm{Si}$ : C, 59.25; $\mathrm{H}$, 6.22. Found: C, 59.09; H, 6.22.

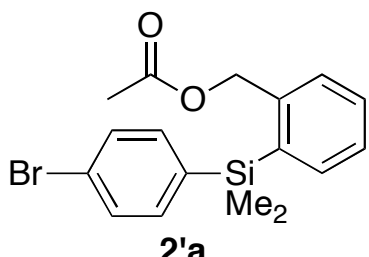

2'a

Preparation of [2-(acetoxymethyl)phenyl](4-bromophenyl)dimethylsilane (2'a). To a solution of (4-bromophenyl)[2-(hydroxymethyl)phenyl]dimethylsilane $(3.2 \mathrm{~g}, 10 \mathrm{mmol})$ in $\mathrm{Et}_{2} \mathrm{O}(20 \mathrm{~mL})$ were added DMAP $(12.2 \mathrm{mg}, 0.10$ $\mathrm{mmol})$, pyridine $(1.58 \mathrm{~g}, 20 \mathrm{mmol})$, and acetyl chloride $(0.86 \mathrm{~g}, 11 \mathrm{mmol})$ at $0{ }^{\circ} \mathrm{C}$. The resulting mixture was stirred at $\mathrm{rt}$ overnight, diluted with $\mathrm{Et}_{2} \mathrm{O}$, neutralized with a $1 \mathrm{M} \mathrm{HCl}$ aqueous solution $(20 \mathrm{~mL})$, and washed with water and brine. The organic layers were dried over anhydrous $\mathrm{MgSO}_{4}$, filtered through a Celite pad, and then concentrated in vacuo. The residue was purified by flash chromatography on silica gel to afford 2'a $(3.7 \mathrm{~g}, 100 \%)$ as a white solid, $\mathrm{mp} 57.4-58.0^{\circ} \mathrm{C}, \mathrm{R}_{\mathrm{f}} 0.44$ (hexane-ethyl acetate $\left.=10: 1\right)$. ${ }^{1} \mathrm{H}$ NMR (400 $\left.\mathrm{MHz}, \mathrm{CDCl}_{3}\right) \delta 7.55(\mathrm{~d}, J=7.3 \mathrm{~Hz}, 1 \mathrm{H}), 7.49-7.28(\mathrm{~m}, 7 \mathrm{H}), 4.94(\mathrm{~s}, 2 \mathrm{H}), 1.92(\mathrm{~s}, 3 \mathrm{H}), 0.59$ (s, 6H); ${ }^{13} \mathrm{C}$ NMR $\left(101 \mathrm{MHz}, \mathrm{CDCl}_{3}\right) \delta 170.4,141.1,137.2,136.4,135.6,135.4,130.9,129.9,129.6,127.6$, 123.9, 66.5, 20.9, -1.0; IR (KBr): 2959, 1730, 1566, 1481, 1377, 1259, 1244, 1069, 1034, 1011, 837, 824, 800, 762, 721, $494 \mathrm{~cm}^{-1}$; Anal. Calcd for $\mathrm{C}_{17} \mathrm{H}_{19} \mathrm{BrO}_{2} \mathrm{Si}: \mathrm{C}, 56.20 ; \mathrm{H}, 5.27$. Found: C, 56.19; H, 5.22 .

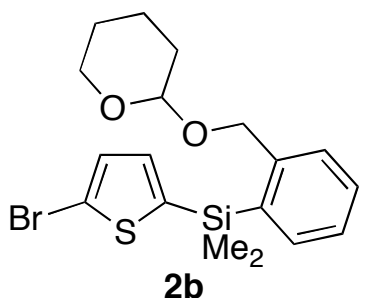

Preparation of (2-bromo-5-thienyl)dimethyl[2-(tetrahydro-2H-pyranoxymethyl)phenyl]silane (2b). $\quad$ To $\quad$ a solution of dimethyl[2-(tetrahydro-2H-pyranoxymethyl)phenyl](2-thienyl)silane (8.4 g, 25 $\mathrm{mmol})$ in $\mathrm{Et}_{2} \mathrm{O}(25 \mathrm{~mL})$ were added TMEDA $(3.4 \mathrm{~g}, 29 \mathrm{mmol})$ and a $1.6 \mathrm{M}$ solution of $n$-BuLi $(28 \mathrm{mmol})$ in hexane at $-40{ }^{\circ} \mathrm{C}$, after being stirred at $\mathrm{rt}$ for 2 h, 1,2-dibromo-1,1,2,2-tetrafluoroethane $(7.8 \mathrm{~g}, 30 \mathrm{mmol})$ was added at $-40{ }^{\circ} \mathrm{C}$, and the resulting mixture was stirred at the same temperature for further $1 \mathrm{~h}$, quenched with a saturated $\mathrm{Na}_{2} \mathrm{~S}_{2} \mathrm{O}_{3}$ aqueous solution $(100 \mathrm{~mL})$, and then diluted with diethyl ether (300 $\mathrm{mL}$ ). The organic layers were dried over anhydrous $\mathrm{MgSO}_{4}$ and concentrated in vacuo. The residue was purified by flash chromatography on silica gel to afford $\mathbf{2 b}(9.3 \mathrm{~g}, 90 \%)$ as a yellowish oil, $\mathrm{R}_{\mathrm{f}} 0.27$ (hexane-ethyl acetate = 15:1). ${ }^{1} \mathrm{H} \mathrm{NMR}\left(400 \mathrm{MHz}, \mathrm{CDCl}_{3}\right) \delta 7.49(\mathrm{t}, J=7.6 \mathrm{~Hz}, 2 \mathrm{H}), 7.41(\mathrm{td}, J=7.5$, $1.0 \mathrm{~Hz}, 1 \mathrm{H}), 7.27(\mathrm{t}, J=7.3 \mathrm{~Hz}, 1 \mathrm{H}), 7.06(\mathrm{~d}, J=3.5 \mathrm{~Hz}, 1 \mathrm{H}), 6.97(\mathrm{~d}, J=3.5 \mathrm{~Hz}, 1 \mathrm{H}), 4.73(\mathrm{~d}, J=$ $12.1 \mathrm{~Hz}, 1 \mathrm{H}), 4.54(\mathrm{t}, J=3.5 \mathrm{~Hz}, 1 \mathrm{H}), 4.46(\mathrm{~d}, J=12.1 \mathrm{~Hz}, 1 \mathrm{H}), 3.87-3.75(\mathrm{~m}, 1 \mathrm{H}), 3.52-3.42(\mathrm{~m}, 1 \mathrm{H})$, $1.88-1.75(\mathrm{~m}, 1 \mathrm{H}), 1.72-1.41(\mathrm{~m}, 5 \mathrm{H}), 0.62(\mathrm{~s}, 6 \mathrm{H}) ;{ }^{13} \mathrm{C} \mathrm{NMR}\left(101 \mathrm{MHz}, \mathrm{CDCl}_{3}\right) \delta 143.9,141.6,135.5$, 
$135.2,134.9,131.1,129.9,128.7,126.9,117.3,97.8,68.7,62.2,30.6,25.6,19.5,0.2,0.1 \mathrm{~cm}^{-1}$; IR (neat): 2943, 2870, 1439, 1406, 1350, 1259, 1204, 1119, 1078, 1026, 974, 953, 907, 870, 837, 812, 779, 754, 691, $656 \mathrm{~cm}^{-1}$; Anal. Calcd for $\mathrm{C}_{18} \mathrm{H}_{23} \mathrm{BrO}_{2} \mathrm{SSi}$ : C, 52.55; H, 5.63. Found: C, 52.72; H, 5.63.

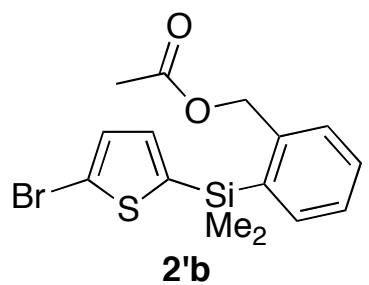
was added at the same temperature, and the resulting mixture was stirred at rt overnight. Acetyl chloride $(0.39 \mathrm{~g}, 5.0 \mathrm{mmol})$ was added at $0{ }^{\circ} \mathrm{C}$, and then the resulting mixture was stirred at $\mathrm{rt}$ for additional $6 \mathrm{~h}$. The mixture was diluted with diethyl ether, washed with a saturated $\mathrm{NaHCO}_{3}$ aqueous solution, water, and brine, and the organic layers were dried over anhydrous $\mathrm{MgSO}_{4}$. After concentration in vacuo, the residue was purified by flash chromatography on silica gel to afford $\mathbf{2 b}$ $(1.14 \mathrm{~g}, 62 \%)$ as a yellowish oil, $\mathrm{R}_{\mathrm{f}} 0.29$ (hexane-ethyl acetate $\left.=10: 1\right) .{ }^{1} \mathrm{H}$ NMR $\left(400 \mathrm{MHz}, \mathrm{CDCl}_{3}\right) \delta$ $7.57(\mathrm{~d}, J=7.3 \mathrm{~Hz}, 1 \mathrm{H}), 7.48-7.31(\mathrm{~m}, 3 \mathrm{H}), 7.09(\mathrm{~d}, J=3.5 \mathrm{~Hz}, 1 \mathrm{H}), 6.99(\mathrm{~d}, J=3.7 \mathrm{~Hz}, 1 \mathrm{H}), 5.07(\mathrm{~s}$, 2H), $2.02(\mathrm{~s}, 3 \mathrm{H}), 0.65(\mathrm{~s}, 6 \mathrm{H}) ;{ }^{13} \mathrm{C} \mathrm{NMR}\left(101 \mathrm{MHz}, \mathrm{CDCl}_{3}\right) \delta 170.4,141.0,135.9,135.6,135.4,131.2$, 130.1, 129.6, 127.7, 117.6, 66.5, 21.0, $0.1 \mathrm{~cm}^{-1}$; IR (neat): 3057, 2957, 1736, 1437, 1404, 1379, 1362, 1286, 1227, 1128, 1078, 1069, 1026, 999, 955, 837, 810, 779, 756, 692, $656 \mathrm{~cm}^{-1}$; Anal. Calcd for $\mathrm{C}_{15} \mathrm{H}_{17} \mathrm{BrO}_{2} \mathrm{SSi}: \mathrm{C}, 48.78$; H, 4.64. Found: C, 48.72; H, 4.67.

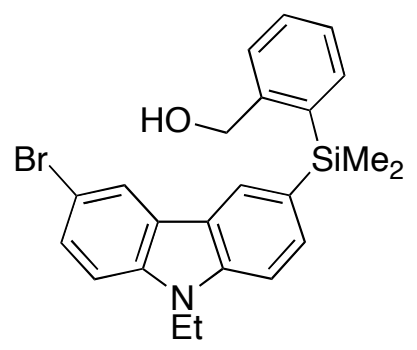

Preparation of (3-bromo-9-ethyl-9H-6-carbazolyl)[2-(hydroxymethyl)phenyl]dimethylsilane. To a solution of 3,6-dibromo-9-ethyl-9H-carbazole ${ }^{5}$ $(7.7 \mathrm{~g}, 22 \mathrm{mmol})$ in THF $(60 \mathrm{~mL})$ was added a $1.6 \mathrm{M}$ solution of $n$-BuLi in hexane $(14 \mathrm{~mL}, 22 \mathrm{mmol})$ over $30 \mathrm{~min}$ at $-78{ }^{\circ} \mathrm{C}$, and the resulting mixture was stirred at $-78^{\circ} \mathrm{C}$ for $1 \mathrm{~h}$. To this was added $4(3.6 \mathrm{~g}, 22 \mathrm{mmol})$ at $-78^{\circ} \mathrm{C}$, and the resulting mixture was warmed slowly to rt, stirred overnight, and then quenched with a saturated $\mathrm{NH}_{4} \mathrm{Cl}$ aqueous solution at $0{ }^{\circ} \mathrm{C}$. The aqueous layer was extracted with diethyl ether, and the combined organic layers were dried over anhydrous $\mathrm{MgSO}_{4}$. After concentration in vacuo, the residue was purified by flash chromatography on silica gel to afford the title compound $(8.6 \mathrm{~g}, 90 \%)$ as a white solid, $\mathrm{mp}$ 49.2-51.0 ${ }^{\circ} \mathrm{C}, \mathrm{R}_{\mathrm{f}} 0.37$ (hexane-ethyl acetate = 2:1). ${ }^{1} \mathrm{H}$ NMR $\left(400 \mathrm{MHz}, \mathrm{CDCl}_{3}\right) \delta 8.18(\mathrm{~s}, 2 \mathrm{H}), 7.63(\mathrm{~d}$, $J=7.5 \mathrm{~Hz}, 1 \mathrm{H}), 7.56(\mathrm{~d}, J=8.1 \mathrm{~Hz}, 1 \mathrm{H}), 7.52(\mathrm{dd}, J=8.6,1.6 \mathrm{~Hz}, 1 \mathrm{H}), 7.48-7.22(\mathrm{~m}, 4 \mathrm{H}), 4.55(\mathrm{~d}, J$ $=4.4 \mathrm{~Hz}, 2 \mathrm{H}), 4.32(\mathrm{q}, J=7.1 \mathrm{~Hz}, 2 \mathrm{H}), 1.41(\mathrm{t}, J=7.1 \mathrm{~Hz}, 3 \mathrm{H}), 1.24(\mathrm{br}, J=5.3 \mathrm{~Hz}, 1 \mathrm{H}), 0.69(\mathrm{~s}, 6 \mathrm{H})$; ${ }^{13} \mathrm{C}$ NMR $\left(101 \mathrm{MHz}, \mathrm{CDCl}_{3}\right) \delta 146.4,140.8,138.4,136.3,135.4,131.5,129.8,128.3,128.1,127.7$, $126.9,126.3,124.3,123.1,121.9,111.8,109.8,108.6,65.3,37.8,13.9,0.2$; IR (KBr): 3418, 2972, 2951, 1587, 1474, 1435, 1346, 1286, 1275, 1232, 1157, 1097, 847, 802, 773, $754 \mathrm{~cm}^{-1}$; HRMS (FAB+) Calcd for $\mathrm{C}_{23} \mathrm{H}_{24}$ BrNOSi: $\mathrm{M}^{+}$, 437.0811. Found: $\mathrm{m} / z$ 437.0807.

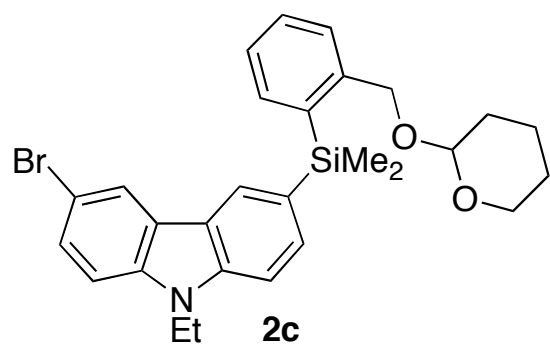

Preparation of (3-bromo-9-ethyl-9H-6-carbazolyl)dimethyl[2(tetrahydro-2H-pyranoxymethyl)phenyl]silane (2c). To a mixture of (3-bromo-9-ethyl-9H-6-carbazolyl)[2-(hydroxymethyl)phenyl]dimethylsilane $(5.6 \mathrm{~g}, 13 \mathrm{mmol})$ and 3,4-dihydro- $2 \mathrm{H}$-pyran $(1.29 \mathrm{~g}$, $15.4 \mathrm{mmol}$ ) were added 3 drops of conc. $\mathrm{HCl}$ at $\mathrm{rt}$, and the resulting mixture was stirred at $\mathrm{rt}$ overnight. The mixture was diluted with $\mathrm{Et}_{2} \mathrm{O}$, neutralized with $\mathrm{NaHCO}_{3}$, dried over anhydrous $\mathrm{MgSO}_{4}$, and 
filtered through a Celite pad. After concentration in vacuo, the residue was purified by flash chromatography on silica gel to afford $2 \mathrm{c}(6.1 \mathrm{~g}, 91 \%)$ as a colorless viscous oil, $\mathrm{R}_{\mathrm{f}} 0.19$ (hexane-ethyl acetate = 15:1). ${ }^{1} \mathrm{H}$ NMR $\left(400 \mathrm{MHz}, \mathrm{CDCl}_{3}\right) \delta 8.18(\mathrm{~s}, 1 \mathrm{H}), 8.17(\mathrm{~s}, 1 \mathrm{H}), 7.60-7.46(\mathrm{~m}, 4 \mathrm{H}), 7.41(\mathrm{td}, J$ $=7.4,1.4 \mathrm{~Hz}, 1 \mathrm{H}), 7.36(\mathrm{~d}, J=8.1 \mathrm{~Hz}, 1 \mathrm{H}), 7.32-7.23(\mathrm{~m}, 2 \mathrm{H}), 4.67(\mathrm{~d}, J=11.9 \mathrm{~Hz}, 1 \mathrm{H}), 4.44-4.37$ $(\mathrm{m}, 2 \mathrm{H}), 4.32(\mathrm{q}, J=7.1 \mathrm{~Hz}, 2 \mathrm{H}), 3.73-3.64(\mathrm{~m}, 1 \mathrm{H}), 3.35-3.26(\mathrm{~m}, 1 \mathrm{H}), 1.80-1.67(\mathrm{~m}, 1 \mathrm{H}), 1.62-1.31$ $(\mathrm{m}, 8 \mathrm{H}), 0.68(\mathrm{~s}, 3 \mathrm{H}), 0.67(\mathrm{~s}, 3 \mathrm{H}) ;{ }^{13} \mathrm{C} \mathrm{NMR}\left(101 \mathrm{MHz}, \mathrm{CDCl}_{3}\right) \delta$ 144.0, 140.7, 138.3, 136.6, 135.4, $131.8,129.5,128.5,128.1,127.8,126.7,126.5,124.4,123.1,121.7,111.7,109.7,108.3,97.8,68.8$, 62.0, 37.7, 30.5, 25.5, 19.4, 13.9, -0.3, -0.4; IR (neat): 3053, 3009, 2947, 2872, 1620, 1589, 1564, $1470,1435,1381,1346,1286,1275,1259,1232,1200,1182,1157,1128,1097,1078,1053,1024,976$, 907, 870, 847, 800, 754, 689, 667, 635, $569 \mathrm{~cm}^{-1}$; Anal. Calcd for $\mathrm{C}_{28} \mathrm{H}_{32} \mathrm{BrNO}_{2} \mathrm{Si}$ : C, 64.36; H, 6.17. Found: C, 64.10; H, 6.05.

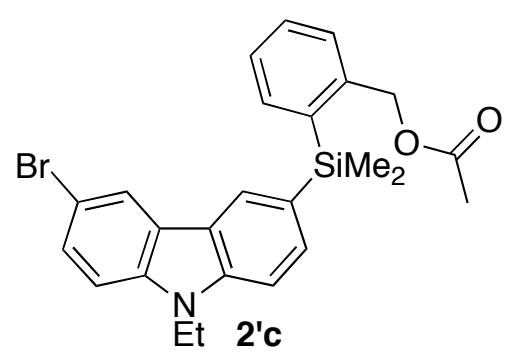

Preparation of [2-(acetoxymethyl)phenyl](3-bromo-9-ethyl-9H-6carbazolyl)dimethylsilane (2'c). To a solution of 3,6-dibromo-9-ethyl-9H-carbazole $(7.0 \mathrm{~g}, 20 \mathrm{mmol})$ in THF $(60 \mathrm{~mL})$ was added a $1.6 \mathrm{M}$ solution of $n$-BuLi in hexane $(12.5 \mathrm{~mL}, 20 \mathrm{mmol})$ over 30 min at $-78{ }^{\circ} \mathrm{C}$, and the resulting mixture was stirred at $-78{ }^{\circ} \mathrm{C}$ for $1 \mathrm{~h}$. To this was added $4(3.3 \mathrm{~g}, 20 \mathrm{mmol})$ at $-78^{\circ} \mathrm{C}$. The resulting mixture was warmed slowly to $\mathrm{rt}$ and stirred for further $17 \mathrm{~h}$. Acetyl chloride $(1.6 \mathrm{~g}, 20 \mathrm{mmol})$ was added at $0{ }^{\circ} \mathrm{C}$, and the resulting mixture was stirred at $\mathrm{rt}$ for $6 \mathrm{~h}$. The reaction mixture was diluted with $\mathrm{Et}_{2} \mathrm{O}$ and washed with a saturated $\mathrm{NaHCO}_{3}$ aqueous solution, water, and then brine. The organic layers were dried over anhydrous $\mathrm{MgSO}_{4}$ before concentration in vacuo, and the residue was purified by flash chromatography on silica gel to afford 2'c $(8.8 \mathrm{~g}, 92 \%)$ as a colorless viscous oil, $\mathrm{R}_{\mathrm{f}} 0.44$ (hexane-ethyl acetate $\left.=5: 1\right)$. ${ }^{1} \mathrm{H}$ NMR $\left(400 \mathrm{MHz}, \mathrm{CDCl}_{3}\right) \delta 8.17(\mathrm{~d}, J=1.6 \mathrm{~Hz}, 1 \mathrm{H}), 8.14(\mathrm{~s}, 1 \mathrm{H}), 7.62(\mathrm{~d}, J=7.3 \mathrm{~Hz}, 1 \mathrm{H}), 7.56(\mathrm{~d}, J=8.2 \mathrm{~Hz}$, $1 \mathrm{H}), 7.51(\mathrm{dd}, J=8.5,1.8 \mathrm{~Hz}, 1 \mathrm{H}), 7.45-7.32(\mathrm{~m}, 4 \mathrm{H}), 7.28-7.23(\mathrm{~m}, 1 \mathrm{H}), 4.99(\mathrm{~s}, 2 \mathrm{H}), 4.32(\mathrm{q}, J=$ $7.1 \mathrm{~Hz}, 2 \mathrm{H}), 1.84(\mathrm{~s}, 3 \mathrm{H}), 1.41(\mathrm{t}, J=7.1 \mathrm{~Hz}, 3 \mathrm{H}), 0.69(\mathrm{~s}, 6 \mathrm{H}) ;{ }^{13} \mathrm{C} \mathrm{NMR}\left(101 \mathrm{MHz}, \mathrm{CDCl}_{3}\right) \delta 170.4$, $141.2,140.8,138.3,137.7,135.7,131.6,129.6,129.4,128.2,127.5,127.3,126.5,124.4,123.1,121.8$, 111.7, 109.8, 108.5, 66.6, 37.7, 20.9, 13.9, -0.4; IR (neat): 3055, 2974, 2897, 1732, 1622, 1589, 1474, 1435, 1379, 1346, 1286, 1275, 1232, 1157, 1128, 1097, 1024, 849, 802, 773, 756, 691, $635 \mathrm{~cm}^{-1}$; Anal. Calcd for $\mathrm{C}_{25} \mathrm{H}_{26} \mathrm{BrNO}_{2} \mathrm{Si}$ : C, 62.49; H, 5.45. Found: C, 62.79; H, 5.44.

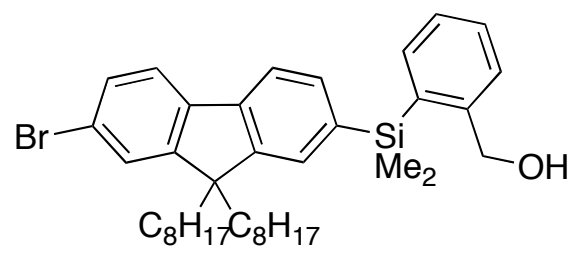

Preparation of (2-bromo-9,9'-dioctyl-9H-7-fluorenyl)[2-(hydroxymethyl)phenyl]dimethylsilane. To a solution of 2,7-dibromo-9,9'-dioctyl-9H-fluorene (22 g, $40 \mathrm{mmol})$ in THF $(120 \mathrm{~mL})$ was added a $1.6 \mathrm{M}$ solution of $n$-BuLi in hexane $(25 \mathrm{~mL}$, $40 \mathrm{mmol}$ ) over $30 \mathrm{~min}$ at $-78^{\circ} \mathrm{C}$, and the resulting mixture was stirred at $-78{ }^{\circ} \mathrm{C}$ for $1 \mathrm{~h}$. To this was added 4 (6.6 g, $\left.40 \mathrm{mmol}\right)$ at $-78{ }^{\circ} \mathrm{C}$, and the resulting mixture was warmed slowly to $\mathrm{rt}$, stirred overnight, and then quenched with a saturated $\mathrm{NH}_{4} \mathrm{Cl}$ aqueous solution at $0{ }^{\circ} \mathrm{C}$. The aqueous layer was extracted with diethyl ether, and the combined organic layers were dried over anhydrous $\mathrm{MgSO}_{4}$. After concentration in vacuo, the residue was purified by flash chromatography on silica gel to afford the title compound (25 $\mathrm{g}, 100 \%)$ as a colorless viscous oil, $\mathrm{R}_{\mathrm{f}} 0.25$ (hexane-ethyl acetate $\left.=10: 1\right) .{ }^{1} \mathrm{H}$ NMR $\left(400 \mathrm{MHz}, \mathrm{CDCl}_{3}\right) \delta 7.63(\mathrm{~d}, J=$ $7.5 \mathrm{~Hz}, 1 \mathrm{H}), 7.57-7.50(\mathrm{~m}, 2 \mathrm{H}), 7.48-7.38(\mathrm{~m}, 6 \mathrm{H}), 7.28(\mathrm{td}, J=7.1,2.0 \mathrm{~Hz}, 1 \mathrm{H}), 4.52(\mathrm{~d}, J=5.7 \mathrm{~Hz}$, 2H), 1.97-1.80 (m, 4H), 1.30-0.94 (m, 21H), $0.83(\mathrm{t}, J=7.1 \mathrm{~Hz}, 6 \mathrm{H}), 0.68-0.48(\mathrm{~m}, 4 \mathrm{H}), 0.65(\mathrm{~s}, 6 \mathrm{H})$; ${ }^{13} \mathrm{C}$ NMR $\left(101 \mathrm{MHz}, \mathrm{CDCl}_{3}\right) \delta 152.9,149.6,146.4,141.0,139.7,137.8,136.2,135.5,132.5,129.81$, $129.79,128.1,128.0,126.9,126.1,121.2,121.1,119.3,65.3,55.4,40.1,31.9,30.0,29.28,29.25,23.8$, 22.7, 14.2, -0.7; IR (neat): 3393, 3053, 2955, 2926, 2855, 1601, 1560, 1454, 1396, 1377, 1256, 1200, 
1126, 1092, 1078, 1061, 1003, 876, 814, 777, 752, 689, $646 \mathrm{~cm}^{-1}$; Anal. Calcd for $\mathrm{C}_{38} \mathrm{H}_{53} \mathrm{BrOSi}$ : C, 72.01; H, 8.43. Found: C, 71.90; H, 8.39.

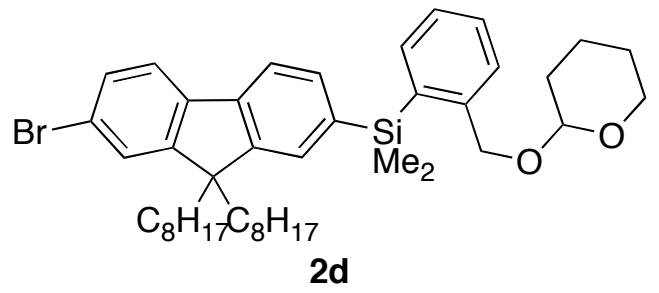

was diluted with $\mathrm{Et}_{2} \mathrm{O}$, neutralized with $\mathrm{NaHCO}_{3}$, dried over anhydrous $\mathrm{MgSO}_{4}$, and filtered through Celite pad. After concentration in vacuo, the residue was purified by flash chromatography on silica gel to afford $2 \mathbf{d}(22 \mathrm{~g}, 95 \%)$ as a colorless viscous oil, $\mathrm{R}_{\mathrm{f}} 0.34$ (hexane-ethyl acetate $\left.=20: 1\right)$. ${ }^{1} \mathrm{H}$ NMR (400 $\left.\mathrm{MHz}, \mathrm{CDCl}_{3}\right) \delta 7.62(\mathrm{~d}, J=7.3 \mathrm{~Hz}, 1 \mathrm{H}), 7.54(\mathrm{~d}, J=8.6 \mathrm{~Hz}, 1 \mathrm{H}), 7.50-7.35(\mathrm{~m}, 7 \mathrm{H}), 7.26-7.20(\mathrm{~m}$, $1 \mathrm{H}), 4.66(\mathrm{~d}, J=12.1 \mathrm{~Hz}, 1 \mathrm{H}), 4.50(\mathrm{t}, J=3.2 \mathrm{~Hz}, 1 \mathrm{H}), 4.40(\mathrm{~d}, J=12.1 \mathrm{~Hz}, 1 \mathrm{H}), 3.84-3.74(\mathrm{~m}, 1 \mathrm{H})$, 3.47-3.39 (m, 1H), 1.96-1.75 (m, 5H), 1.69-1.41 (m, 5H), 1.28-0.94 (m, 20H), 0.83 (t, J=7.2 Hz, 6H), $0.68-0.52(\mathrm{~m}, 4 \mathrm{H}), 0.63(\mathrm{~s}, 6 \mathrm{H}) ;{ }^{13} \mathrm{C} \mathrm{NMR}\left(101 \mathrm{MHz}, \mathrm{CDCl}_{3}\right) \delta$ 153.0, 149.4, 144.0, 140.7, 139.8, 137.8, 136.4, 135.5, 132.7, 129.7, 129.4, 128.4, 128.2, 126.6, 126.1, 121.0, 119.1, 109.7, 97.6, 68.8, $61.9,55.4,40.1,31.9,30.6,30.0,29.31,29.28,25.6,23.9,22.7,19.4,14.2,-0.65,-0.73$; IR (neat) 2926, 2853, 1452, 1258, 1200, 1119, 1092, 1078, 1055, 1026, 1003, 836, 814, 777, 752, $646 \mathrm{~cm}^{-1}$; Anal. Calcd for $\mathrm{C}_{43} \mathrm{H}_{61} \mathrm{BrO}_{2} \mathrm{Si} ; \mathrm{C}, 71.94 ; \mathrm{H}, 8.56$. Found: C, 71.96; H, 8.73.

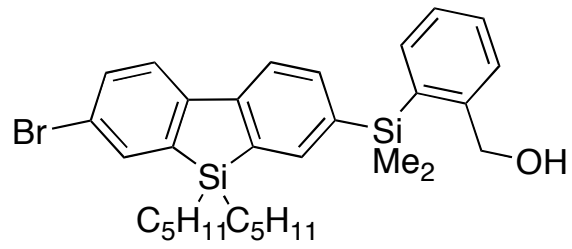

Preparation of (2-bromo-9,9'-dipentyl-9H-9-silafluoren-7-yl)[2(hydroxymethyl)phenyl]dimethylsilane. To a solution of 2,7-dibromo-9,9'-dioctyl-9H-9-silafluorene ${ }^{6}(2.5 \mathrm{~g}, 5.2 \mathrm{mmol})$ in THF $(16 \mathrm{~mL})$ was added a $1.6 \mathrm{M}$ solution of $n$-BuLi in hexane (3.3 $\mathrm{mL}, 5.2 \mathrm{mmol}$ ) over $30 \mathrm{~min}$ at $-78{ }^{\circ} \mathrm{C}$, and the resulting mixture was stirred at $-78{ }^{\circ} \mathrm{C}$ for $1 \mathrm{~h}$. To this was added $4(0.85 \mathrm{~g}, 5.2$ mmol) at $-78{ }^{\circ} \mathrm{C}$. The resulting mixture was warmed slowly to rt, stirred overnight, and the quenched with a saturated $\mathrm{NH}_{4} \mathrm{Cl}$ aqueous solution at $0{ }^{\circ} \mathrm{C}$. The aqueous layer was extracted with diethyl ether, and the combined organic layers were dried over anhydrous $\mathrm{MgSO}_{4}$. After concentration in vacuo, the residue was purified by flash chromatography on silica gel to afford the title compound $(1.79 \mathrm{~g}, 61 \%)$ as a viscous oil, $\mathrm{R}_{\mathrm{f}} 0.32$ (hexane-ethyl acetate $\left.=5: 1\right) .{ }^{1} \mathrm{H}$ NMR $\left(400 \mathrm{MHz}, \mathrm{CDCl}_{3}\right) \delta 7.70(\mathrm{~d}, J=8.2 \mathrm{~Hz}$, $2 \mathrm{H}), 7.63(\mathrm{~d}, J=8.4 \mathrm{~Hz}, 2 \mathrm{H}), 7.57-7.46(\mathrm{~m}, 3 \mathrm{H}), 7.45-7.36(\mathrm{~m}, 2 \mathrm{H}), 7.28(\mathrm{t}, J=7.2 \mathrm{~Hz}, 1 \mathrm{H}), 4.53(\mathrm{~d}$, $J=7.5 \mathrm{~Hz}, 2 \mathrm{H}), 1.34-1.13(\mathrm{~m}, 13 \mathrm{H}), 0.92-0.84(\mathrm{~m}, 4 \mathrm{H}), 0.77(\mathrm{t}, J=6.8 \mathrm{~Hz}, 6 \mathrm{H}), 0.61(\mathrm{~s}, 6 \mathrm{H}){ }^{13} \mathrm{C}$ NMR (101 MHz, $\left.\mathrm{CDCl}_{3}\right) \delta 148.1,146.6,146.4,141.0,138.6,137.7,136.8,135.86,135.83,135.6$, 135.4, 132.7, 129.8, 128.1, 126.9, 122.5, 122.0, 120.3, 65.4, 35.5, 23.6, 22.2, 14.1, 12.1, -0.8; IR (neat): 3377, 3057, 2955, 2922, 2870, 2856, 1582, 1458, 1443, 1379, 1366, 1250, 1113, 1076, 999, 837, $827,775,750,646 \mathrm{~cm}^{-1}$; Anal. Calcd for $\mathrm{C}_{31} \mathrm{H}_{41} \mathrm{BrOSi}_{2}$; C, 65.81; H, 7.30. Found: C, 65.94; H, 7.51.

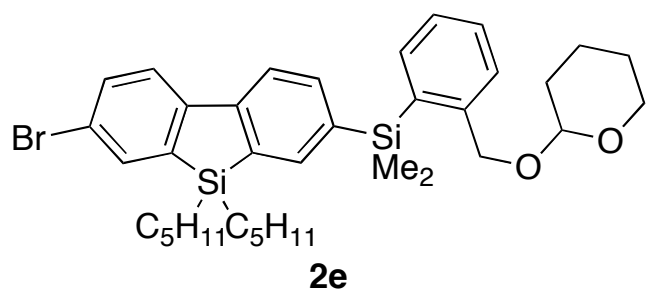

Preparation of (2-bromo-9,9'-dipentyl-9H-9-silafluoren-7yl)dimethyl[2-(tetrahydro-2H-pyranoxymethyl)phenyl]sila ne (2e). To a solution of (2-bromo-9,9'-dipentyl-9H-9-silafluoren-7-yl)[2-(hydroxymethyl)phenyl]dimethylsilane (1.36 g, $2.4 \mathrm{mmol})$ and 3,4-dihydro- $2 \mathrm{H}$-pyran $(0.4 \mathrm{~g}, 4.8 \mathrm{mmol})$ in $\mathrm{Et}_{2} \mathrm{O}(2.4 \mathrm{~mL})$ were added 2 drops of conc. $\mathrm{HCl}$ at $\mathrm{rt}$, and the resulting mixture was stirred at $\mathrm{rt}$ overnight. The mixture was diluted with $\mathrm{Et}_{2} \mathrm{O}$, neutralized with $\mathrm{NaHCO}_{3}$, dried over anhydrous $\mathrm{MgSO}_{4}$, and filtered through a 
Celite pad. After concentration in vacuo, the residue was purified by flash chromatography on silica gel to afford $2 \mathbf{e}(1.47 \mathrm{~g}, 94 \%)$ as a colorless oil, $\mathrm{R}_{\mathrm{f}} 0.35$ (hexane-ethyl acetate $\left.=20: 1\right)$. ${ }^{1} \mathrm{H} \mathrm{NMR}(400 \mathrm{MHz}$, $\left.\mathrm{CDCl}_{3}\right) \delta$ 7.75-7.62 (m, 4H), 7.57-7.44 (m, 4H), $7.39(\mathrm{t}, J=7.4 \mathrm{~Hz}, 1 \mathrm{H}), 7.30-7.22(\mathrm{~m}, 1 \mathrm{H}), 4.67(\mathrm{~d}, J$ $=12.1 \mathrm{~Hz}, 1 \mathrm{H}), 4.47(\mathrm{t}, J=3.2 \mathrm{~Hz}, 1 \mathrm{H}), .4 .41(\mathrm{~d}, J=12.1 \mathrm{~Hz}, 1 \mathrm{H}), 3.80-3.70(\mathrm{~m}, 1 \mathrm{H}), 3.44-3.25(\mathrm{~m}$, $1 \mathrm{H}), 1.84-1.70(\mathrm{~m}, 1 \mathrm{H}), 1.66-1.02(\mathrm{~m}, 17 \mathrm{H}), 0.95-0.85(\mathrm{~m}, 6 \mathrm{H}), 0.84-0.74(\mathrm{~m}, 4 \mathrm{H}), 0.620(\mathrm{~s}, 3 \mathrm{H})$, $0.617(\mathrm{~s}, 3 \mathrm{H}) ;{ }^{13} \mathrm{C}$ NMR $\left(101 \mathrm{MHz}, \mathrm{CDCl}_{3}\right) \delta 147.8,146.8,144.0,141.1,138.7,137.8,136.4,136.1$, 136.0, 135.6, 135.4, 132.7, 129.5, 128.5, 126.7, 122.4, 121.9, 120.0, 97.7 68.8, 61.9, 35.6, 30.6, 25.5, 23.6, 22.2, 19.4, 14.1, 12.2, -0.7, -0.8; IR (neat): 2955, 2922, 2870, 2856, 1441, 1250, 1200, 1115, 1078, 1026, 837, 816, 775, $750 \mathrm{~cm}^{-1}$; Anal. Calcd for $\mathrm{C}_{36} \mathrm{H}_{49} \mathrm{BrO}_{2} \mathrm{Si}_{2} ; \mathrm{C}, 66.54 ; \mathrm{H}, 7.60$. Found: C, $66.39 ; \mathrm{H}, 7.42$.

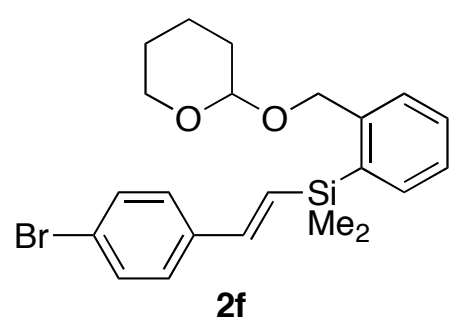

\section{(E)-2-[(4-Bromophenyl)ethenyl][2-(tetrahydro-2H-pyranoxymethyl)-} phenyl]dimethylsilane (2f). To a solution of dimethyl[2-(tetrahydro- $2 \mathrm{H}$ pyranoxymethyl)phenyl]silane $(0.79 \quad \mathrm{~g}, \quad 3.2 \quad \mathrm{mmol})$ and 1-bromo-4-ethynylbenzene $(0.54 \mathrm{~g}, 3.0 \mathrm{mmol})$ in hexane $(0.3 \mathrm{~mL})$ were slowly added a $10 \mathrm{wt} \%$ hexane solution of $t-\mathrm{Bu}_{3} \mathrm{P}(6.1 \mathrm{mg}, 3.0 \mu \mathrm{mol})$ and a $0.01 \mathrm{M}$ solution of platinum(0)-1,3-divinyl-1,1,3,3-tetramethyldisiloxane $(0.3 \mathrm{~mL}, 3.0 \mu \mathrm{mol})$ in hexane at $-40{ }^{\circ} \mathrm{C}$ over $30 \mathrm{~min}$. The resulting mixture was stirred at the same temperature for further $30 \mathrm{~min}$ and then at $\mathrm{rt}$ for $23 \mathrm{~h}$, filtered through a Florisil pad. Concentration in vacuo followed by flash chromatography on silica gel gave $\mathbf{2 f}$ (1.23 g, $95 \%$ ) as a yellow oil, $\mathrm{R}_{\mathrm{f}} 0.13$ (hexane-ethyl acetate $\left.=30: 1\right) .{ }^{1} \mathrm{H} \mathrm{NMR}\left(400 \mathrm{MHz}, \mathrm{CDCl}_{3}\right) \delta 7.57(\mathrm{dd}, J$ $=7.4,1.4 \mathrm{~Hz}, 1 \mathrm{H}), 7.50(\mathrm{~d}, J=7.5 \mathrm{~Hz}, 1 \mathrm{H}), 7.47-7.37(\mathrm{~m}, 3 \mathrm{H}), 7.32-7.27(\mathrm{~m}, 3 \mathrm{H}), 6.86(\mathrm{~d}, J=19.0$ $\mathrm{Hz}, 1 \mathrm{H}), 6.65(\mathrm{~d}, J=19.0 \mathrm{~Hz}, 1 \mathrm{H}), 4.86(\mathrm{~d}, J=12.1 \mathrm{~Hz}, 1 \mathrm{H}), 4.65(\mathrm{t}, J=3.6 \mathrm{~Hz}, 1 \mathrm{H}), 4.59(\mathrm{~d}, J=11.9$ $\mathrm{Hz}, 1 \mathrm{H}), 3.98-3.82(\mathrm{~m}, 1 \mathrm{H}), 3.52-3.42(\mathrm{~m}, 1 \mathrm{H}), 1.91-1.77(\mathrm{~m}, 1 \mathrm{H}), 1.76-1.43(\mathrm{~m}, 5 \mathrm{H}), 0.50(\mathrm{~s}, 6 \mathrm{H})$; ${ }^{13} \mathrm{C}$ NMR $\left(101 \mathrm{MHz}, \mathrm{CDCl}_{3}\right) \delta 143.8,143.3,137.0,136.3,134.9,131.4,129.4,128.9,128.4,127.8$, 126.8, 121.8, 97.9, 68.9, 62.2, 30.7, 25.6, 19.5, -1.1, -1.2; IR (neat): 2947, 2870, 1603, 1485, 1439, 1396, 1350, 1248, 1200, 1117, 1074, 1026, 1009, 988, 961, 907, 845, 818, 785, $752 \mathrm{~cm}^{-1}$; Anal. Calcd for $\mathrm{C}_{22} \mathrm{H}_{27} \mathrm{BrO}_{2} \mathrm{Si} ; \mathrm{C}, 61.25 ; \mathrm{H}, 6.31$. Found: $\mathrm{C}, 61.52 ; \mathrm{H}, 6.32$.

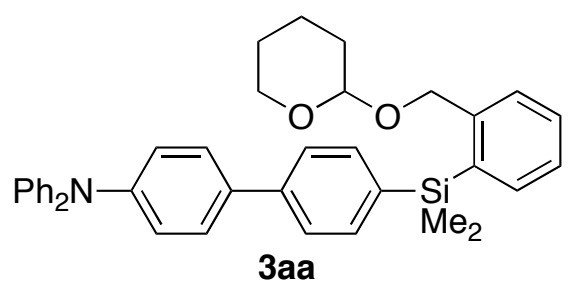

Gram-scale Cross-coupling reaction of 1a with 2a. To a solution of $1 \mathrm{a}(4.9 \mathrm{~g}, 12 \mathrm{mmol}), \mathrm{K}_{2} \mathrm{CO}_{3}(3.5 \mathrm{~g}, 2.5 \mathrm{mmol}),\left[\left(\eta^{3}-\mathrm{C}_{3} \mathrm{H}_{5}\right) \mathrm{PdCl}\right]_{2}$ (18 mg, $50 \mu \mathrm{mol}$, measured in a glove box), RuPhos (98 mg, 0.21 $\mathrm{mmol})$, and $\mathrm{CuI}(57 \mathrm{mg}, 0.30 \mathrm{mmol})$ in DMF $(8 \mathrm{~mL})$ and THF (22 $\mathrm{mL})$ in a Schlenk tube was added $2 \mathrm{a}(4.1 \mathrm{~g}, 10 \mathrm{mmol})$, and the resulting mixture was stirred at $75^{\circ} \mathrm{C}$ for $7 \mathrm{~h}$. The mixture was filtered through a Florisil pad, diluted with $\mathrm{Et}_{2} \mathrm{O}$, washed with water for 5 times and brine, and dried over anhydrous $\mathrm{MgSO}_{4}$. Concentration in vacuo followed by distillation under vacuum $(3.0 \mathrm{mmHg})$ to gave cyclic silyl ether $4(1.50 \mathrm{~g}, 86 \%$ based on consumed 1a). The residue was further purified by flash chromatography on silica gel (hexane-ethyl acetate $=20: 1$, 10:1, then $2: 1$ as eluents) to give 4'-(diphenylamino)-4-([2-(tetrahydro-2H-pyranoxymethyl)phenyl]dimethylsilyl)biphenyl (3aa, 5.0 g, $88 \%)$ and unreacted $1 \mathrm{a}(0.58 \mathrm{~g}, 12 \%)$. 3aa: A white solid, $\mathrm{mp} 52.6-53.5^{\circ} \mathrm{C}, \mathrm{R}_{\mathrm{f}} 0.18$ (hexane-ethyl acetate = 15:1). ${ }^{1} \mathrm{H}$ NMR $\left(400 \mathrm{MHz}, \mathrm{CDCl}_{3}\right) \delta 7.58-7.37(\mathrm{~m}, 8 \mathrm{H}), 7.34-7.20(\mathrm{~m}, 6 \mathrm{H}), 7.16-7.07(\mathrm{~m}$, $6 \mathrm{H}), 7.02(\mathrm{t}, J=7.3 \mathrm{~Hz}, 2 \mathrm{H}), 4.68(\mathrm{~d}, J=11.9 \mathrm{~Hz}, 1 \mathrm{H}), 4.46-4.37(\mathrm{~m}, 2 \mathrm{H}), 3.80-3.70(\mathrm{~m}, 1 \mathrm{H})$, 3.45-3.35 (m, 1H), 1.84-1.69 (m, 1H), 1.66-1.38 (m, 5H), $0.62(\mathrm{~s}, 3 \mathrm{H}), 0.61(\mathrm{~s}, 3 \mathrm{H}) ;{ }^{13} \mathrm{C}$ NMR $(101$

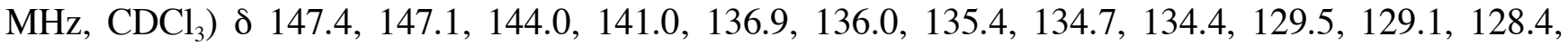
127.6, 126.7, 125.9, 124.4, 123.6, 122.8, 97.8, 68.8, 62.0, 30.6, 25.5, 19.4, -0.7, -0.8; IR (KBr): 3470, 2945, 1589, 1489, 1325, 1277, 1115, 1026, 835, 812, 775, 754, 696, $521 \mathrm{~cm}^{-1}$; Anal. Calcd for 
ja074728s_SI_0824.pdf

$\mathrm{C}_{38} \mathrm{H}_{39} \mathrm{NO}_{2} \mathrm{Si}: \mathrm{C}, 80.10 ; \mathrm{H}, 6.90$. Found: C, 80.07; H, 6.80.

Cross-coupling reaction of 1 with organo[2-(alkoxymethyl)phenyl]dimethylsilanes (2). A general procedure. To a mixture of 1 (1.2-1.5 mmol), $\mathrm{K}_{2} \mathrm{CO}_{3}(0.35 \mathrm{~g}, 2.5 \mathrm{mmol}),\left[\left(\eta^{3}-\mathrm{C}_{3} \mathrm{H}_{5}\right) \mathrm{PdCl}\right]_{2}(1.8 \mathrm{mg}, 5.0 \mu \mathrm{mol}$, measured in a glove box), RuPhos (9.8 mg, $21 \mu \mathrm{mol})$, and CuI $(5.7 \mathrm{mg}, 30 \mu \mathrm{mol})$ in DMF $(0.8 \mathrm{~mL})$ and THF $(2.2 \mathrm{~mL})$ in a Schlenk tube was added $2(1.0 \mathrm{mmol})$, and the resulting mixture was stirred at $75^{\circ} \mathrm{C}$. After the time specified in Table 1, the mixture was filtered through a Florisil pad, diluted with $\mathrm{Et}_{2} \mathrm{O}$, washed with water and brine, and dried over anhydrous $\mathrm{MgSO}_{4}$. Concentration in vacuo followed by flash chromatography on silica gel afforded the corresponding coupling product in a yield listed in Table 1.

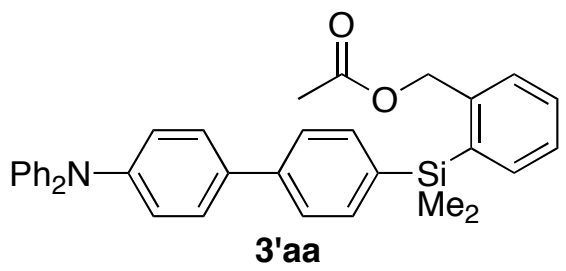

4-([2-Acetoxymethyl]phenyl)dimethylsilyl)-4'-(diphenylamino)biphenyl (3'aa). A white solid, mp $43.4-45.3{ }^{\circ} \mathrm{C}, \mathrm{R}_{\mathrm{f}} 0.26$ (hexane-ethyl acetate $=5: 1) .{ }^{1} \mathrm{H}$ NMR $\left(400 \mathrm{MHz}, \mathrm{CDCl}_{3}\right) \delta 7.60(\mathrm{~d}$, $J=7.1 \mathrm{~Hz}, 1 \mathrm{H}), 7.56-7.31(\mathrm{~m}, 9 \mathrm{H}), 7.29-7.21(\mathrm{~m}, 4 \mathrm{H}), 7.17-7.08$ $(\mathrm{m}, 6 \mathrm{H}), 7.02(\mathrm{t}, J=7.3 \mathrm{~Hz}, 2 \mathrm{H}), 5.00(\mathrm{~s}, 2 \mathrm{H}), 1.91(\mathrm{~s}, 3 \mathrm{H}), 0.63(\mathrm{~s}$, $6 \mathrm{H}) ;{ }^{13} \mathrm{C}$ NMR $\left(101 \mathrm{MHz}, \mathrm{CDCl}_{3}\right) \delta 170.4,147.4,147.2,141.1$, 137.2, 136.4, 135.6, 134.5, 134.3, 129.7, 129.5, 129.1, 127.58, 127.56, 126.0, 124.4, 123.6, 122.9, 66.7, 21.0, -0.8; IR (KBr): 3456, 3057, 3032, 2955, 1736, 1589, 1518, 1489, 1379, 1327, 1317, 1277, 1250, 1234, 1113, 1026, 835, 810, 775, 754, 696, $521 \mathrm{~cm}^{-1}$; Anal. Calcd for $\mathrm{C}_{35} \mathrm{H}_{33} \mathrm{NO}_{2} \mathrm{Si}$ : C, 79.66; H, 6.30. Found: C, 79.48; H, 6.27.

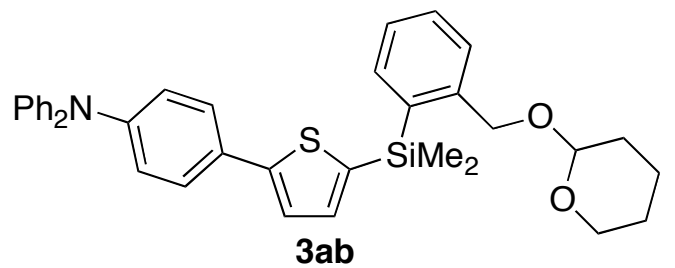

2-(Dimethyl[2-(tetrahydro-2H-pyranoxymethyl)phenyl]sil -yl)-5-[4-(diphenylamino)phenyl]thiophene (3ab). A pale yellow viscous liquid, $\mathrm{R}_{\mathrm{f}} 0.13$ (hexane-ethyl acetate $=20: 1$ ). ${ }^{1} \mathrm{H}$ NMR $\left(400 \mathrm{MHz}, \mathrm{CDCl}_{3}\right) \delta 7.55(\mathrm{dd}, J=7.3,1.1 \mathrm{~Hz}, 1 \mathrm{H})$, $7.53-7.43(\mathrm{~m}, 3 \mathrm{H}), 7.41(\mathrm{td}, J=7.5,1.3 \mathrm{~Hz}, 1 \mathrm{H}), 7.31-7.22$ $(\mathrm{m}, 6 \mathrm{H}), 7.18(\mathrm{~d}, J=3.5 \mathrm{~Hz}, 1 \mathrm{H}), 7.14-7.08(\mathrm{~m}, 4 \mathrm{H})$, $7.07-6.99(\mathrm{~m}, 4 \mathrm{H}), 4.78(\mathrm{~d}, J=11.9 \mathrm{~Hz}, 1 \mathrm{H}), 4.58(\mathrm{t}, J=3.6$

$\mathrm{Hz}, 1 \mathrm{H}), 4.53(\mathrm{~d}, J=12.1 \mathrm{~Hz}, 1 \mathrm{H}), 3.88-3.79(\mathrm{~m}, 1 \mathrm{H}), 3.54-3.42(\mathrm{~m}, 1 \mathrm{H}), 1.90-1.76(\mathrm{~m}, 1 \mathrm{H})$, 1.73-1.44 (m, 5H), $0.67(\mathrm{~s}, 6 \mathrm{H}) ;{ }^{13} \mathrm{C} \mathrm{NMR}\left(101 \mathrm{MHz}, \mathrm{CDCl}_{3}\right) \delta$ 150.0, 147.3, 147.1, 143.9, 137.2, 136.3, 135.7, 135.2, 129.7, 129.1, 128.5, 128.3, 126.8, 126.6, 124.3, 123.54, 123.45, 122.9, 97.8, 68.8, 62.1, 30.6, 25.6, 19.5, 0.3, 0.2; IR (neat): 3055, 2941, 2868,1589, 1529, 1489, 1431, 1327, 1313, 1277 , 1277, 1200, 1117, 1076, 1026, 955, 949, 835, 810, 773, 752, 731, $696 \mathrm{~cm}^{-1}$; HRMS (FAB+) Calcd for $\mathrm{C}_{36} \mathrm{H}_{37} \mathrm{BrNO}_{2} \mathrm{SSi}: \mathrm{M}^{+}$, 575.2314. Found: $m / z$ 575.2327.

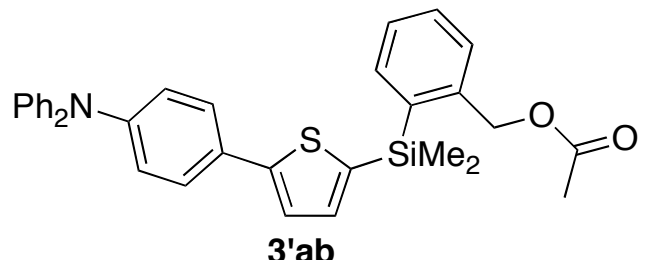
2H), $2.02(\mathrm{~s}, 3 \mathrm{H}), 0.68(\mathrm{~s}, 6 \mathrm{H}) ;{ }^{13} \mathrm{C} \mathrm{NMR}\left(101 \mathrm{MHz}, \mathrm{CDCl}_{3}\right) \delta 170.5,150.3,147.3,147.1,141.1,136.7$, 136.6, 136.3, 135.4, 129.9, 129.5, 129.1, 128.2, 127.6, 126.6, 124.4, 123.5, 122.9, 66.6, 21.1. 0.2; IR (neat) 3057, 3034, 2961, 1738, 1591, 1529, 1495, 1433, 1327, 1315, 1279, 1259, 1234, 1078, 1026, 995, 951, 835, 808, 779, 754, $696 \mathrm{~cm}^{-1}$; HRMS (EI) Calcd for $\mathrm{C}_{33} \mathrm{H}_{31} \mathrm{NO}_{2} \mathrm{SSi}_{2}: \mathrm{M}^{+}, 533.1845$. Found: $\mathrm{m} / \mathrm{z} 533.1846$.
2-([2-(Acetoxymethyl)phenyl]dimethylsilyl)-5-[4-(diphenylamino)phenyl]thiophene (3'ab). A pale yellow viscous oil, $\mathrm{R}_{\mathrm{f}}$ 0.13 (hexane-ethyl acetate $=20: 1) .{ }^{1} \mathrm{H}$ NMR $(400 \mathrm{MHz}$, $\left.\mathrm{CDCl}_{3}\right) \delta 7.61(\mathrm{dd}, J=7.4,0.8 \mathrm{~Hz}, 1 \mathrm{H}), 7.59-7.37(\mathrm{~m}, 5 \mathrm{H})$, $7.34(\mathrm{td}, J=7.1,1.9 \mathrm{~Hz}, 1 \mathrm{H}), 7.29-7.22(\mathrm{~m}, 5 \mathrm{H}), 7.18(\mathrm{~d}, J=$ $3.5 \mathrm{~Hz}, 1 \mathrm{H}), 7.13-7.08(\mathrm{~m}, 4 \mathrm{H}), 7.06-7.00(\mathrm{~m}, 4 \mathrm{H}), 5.10(\mathrm{~s}$, 


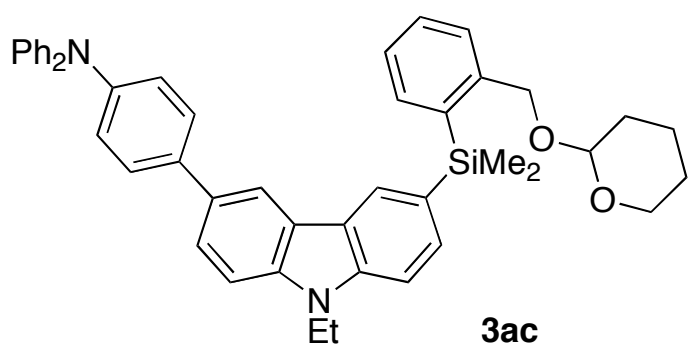

6-[4-(Diphenylamino)phenyl]-3-([2-(tetrahydro-2H-pyranoxymethyl)phenyl]dimethylsilyl)-9-ethyl-9H-carbazole (3ac). A pale yellow viscous oil, $\mathrm{R}_{\mathrm{f}} 0.42$ (hexane-ethyl acetate $=10: 1) .{ }^{1} \mathrm{H}$ NMR $\left(400 \mathrm{MHz}, \mathrm{CDCl}_{3}\right) \delta 8.27(\mathrm{~s}, 1 \mathrm{H})$, $8.25(\mathrm{~d}, J=1.1 \mathrm{~Hz}, 1 \mathrm{H}), 7.67(\mathrm{dd}, J=8.4,1.6 \mathrm{~Hz}, 1 \mathrm{H})$, 7.61-7.53 (m, 4H), $7.49(\mathrm{~d}, J=7.5 \mathrm{~Hz}, 1 \mathrm{H}), 7.46-7.35(\mathrm{~m}$, $3 \mathrm{H}), 7.32-7.22(\mathrm{~m}, 5 \mathrm{H}), 7.20-7.11(\mathrm{~m}, 6 \mathrm{H}), 7.01(\mathrm{t}, J=7.3$ $\mathrm{Hz}, 2 \mathrm{H}), 4.69(\mathrm{~d}, J=12.1 \mathrm{~Hz}, 1 \mathrm{H}), 4.46-4.32(\mathrm{~m}, 4 \mathrm{H})$, 3.74-3.65 (m, 1H), 3.35-3.27 (m, 1H), 1.80-1.67 (m, 1H), 1.62-1.23 (m, 8H), $0.688(\mathrm{~s}, 3 \mathrm{H}), 0.685$ (s, $3 \mathrm{H}) ;{ }^{13} \mathrm{C}$ NMR $\left(101 \mathrm{MHz}, \mathrm{CDCl}_{3}\right) \delta$ 147.6, 146.1, 144.0, 140.9, 139.0, 136.9, 136.4, 135.5, 131.9, 131.3, 129.4, 129.1, 128.4, 127.8, 127.2, 126.6, 126.3, 124.7, 124.4, 124.0, 123.2, 122.8, 122.5, 118.5, 108.5, 108.2, 97.8, 68.9, 62.0, 37.7, 30.6, 25.5, 19.4, 14.0, -0.3, -0.4; IR (neat): 2947, 2870, 1593, $1479,1275,1232,1128,1117,1078,1026,837,806,752,696 \mathrm{~cm}^{-1}$; Anal. Calcd for $\mathrm{C}_{46} \mathrm{H}_{46} \mathrm{~N}_{2} \mathrm{O}_{2} \mathrm{Si}$ : C, 80.43; H, 6.75. Found: C, 80.20; H, 6.77.

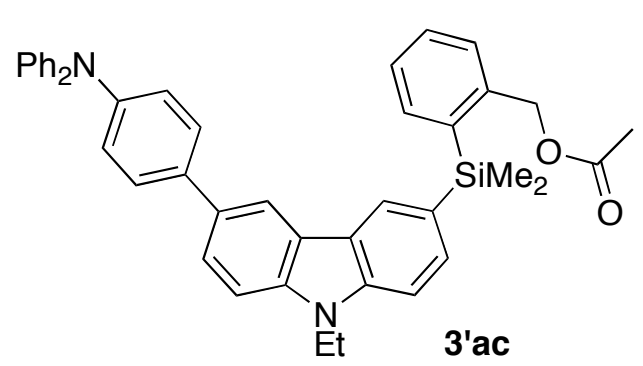

3-([2-(Acetoxymethyl)phenyl]dimethylsilyl)-6-[(4-diphenylamino)phenyl]-9-ethyl-9H-carbazole (3'ac). A white solid, $\mathrm{mp} 75.3-76.4{ }^{\circ} \mathrm{C}, \mathrm{R}_{\mathrm{f}} 0.29$ (hexane-ethyl acetate $=5: 1$ ). ${ }^{1} \mathrm{H}$ $\operatorname{NMR}\left(400 \mathrm{MHz}, \mathrm{CDCl}_{3}\right) \delta 8.25(\mathrm{~d}, J=1.5 \mathrm{~Hz}, 1 \mathrm{H}), 8.24(\mathrm{~s}$, $1 \mathrm{H}), 7.67(\mathrm{dd}, J=8.5,1.7 \mathrm{~Hz}, 1 \mathrm{H}), 7.63(\mathrm{dd}, J=7.2,1.0 \mathrm{~Hz}$, $1 \mathrm{H}), 7.60-7.52(\mathrm{~m}, 3 \mathrm{H}), 7.45-7.31(\mathrm{~m}, 5 \mathrm{H}), 7.30-7.22(\mathrm{~m}, 4 \mathrm{H})$, $7.19-7.11(\mathrm{~m}, 6 \mathrm{H}), 7.01(\mathrm{tt}, J=7.2,1.1 \mathrm{~Hz}, 2 \mathrm{H}), 5.01(\mathrm{~s}, 2 \mathrm{H})$, $4.37(\mathrm{q}, J=7.1 \mathrm{~Hz}, 2 \mathrm{H}), 1.84(\mathrm{~s}, 3 \mathrm{H}), 1.45(\mathrm{t}, J=7.2 \mathrm{~Hz}, 3 \mathrm{H})$, $0.70(\mathrm{~s}, 6 \mathrm{H}) ;{ }^{13} \mathrm{C}$ NMR $\left(101 \mathrm{MHz}, \mathrm{CDCl}_{3}\right) \delta 170.4,147.6,146.1,141.2,141.0,139.0,138.0,136.3$, 135.7, 132.0, 131.1, 129.5, 129.4, 129.1, 127.8, 127.5, 126.7, 126.3, 124.8, 124.4, 124.0, 123.1, 122.9, 122.5, 118.5, 108.5, 108.3, 66.7, 37.7, 21.0, 14.0, -0.4; IR (KBr): 3452, 1736, 1591, 1479, 1277, 1232, 837, 806, 754, $696 \mathrm{~cm}^{-1}$; Anal. Calcd for $\mathrm{C}_{43} \mathrm{H}_{40} \mathrm{~N}_{2} \mathrm{O}_{2} \mathrm{Si}$ : C, 80.09; H, 6.25. Found: C, 80.07; H, 6.34.

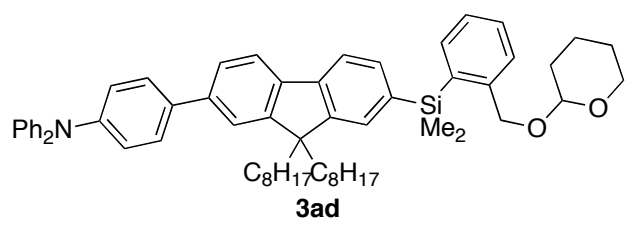

7-[(4-Diphenylamino)phenyl]-2-([2-(tetrahydro-2H-pyranoxymethyl)phenyl]dimethylsilyl)-9,9'-dioctyl-9H-fluorene

(3ad). A pale yellow viscous oil, $\mathrm{R}_{\mathrm{f}} 0.22$ (hexane-ethyl acetate = 9:1). ${ }^{1} \mathrm{H}$ NMR $\left(400 \mathrm{MHz}, \mathrm{CDCl}_{3}\right) \delta 7.72(\mathrm{~d}, J=7.7 \mathrm{~Hz}, 1 \mathrm{H})$, $7.66(\mathrm{~d}, J=7.5 \mathrm{~Hz}, 1 \mathrm{H}), 7.57-7.42(\mathrm{~m}, 8 \mathrm{H}), 7.38(\mathrm{t}, J=7.5 \mathrm{~Hz}$, $1 \mathrm{H}), 7.30-7.20(\mathrm{~m}, 5 \mathrm{H}), 7.14(\mathrm{t}, J=7.4 \mathrm{~Hz}, 6 \mathrm{H}), 7.02(\mathrm{t}, J=7.3 \mathrm{~Hz}, 2 \mathrm{H}), 4.68(\mathrm{~d}, J=12.1 \mathrm{~Hz}, 1 \mathrm{H})$, $4.51(\mathrm{t}, J=3.4 \mathrm{~Hz}, 1 \mathrm{H}), 4.43(\mathrm{~d}, J=12.1 \mathrm{~Hz}, 1 \mathrm{H}), 3.86-3.74(\mathrm{~m}, 1 \mathrm{H}), 3.48-3.39(\mathrm{~m}, 1 \mathrm{H}), 2.00-1.75$ $(\mathrm{m}, 5 \mathrm{H}), 1.70-1.40(\mathrm{~m}, 5 \mathrm{H}), 1.34-0.93(\mathrm{~m}, 20 \mathrm{H}), 0.81(\mathrm{t}, J=7.2 \mathrm{~Hz}, 6 \mathrm{H}), 0.74-0.58(\mathrm{~m}, 10 \mathrm{H}) ;{ }^{13} \mathrm{C}$ NMR (101 MHz, $\left.\mathrm{CDCl}_{3}\right) \delta$ 151.4, 145.0, 147.5, 146.9, 144.0, 141.6, 139.7, 139.5, 137.0, 136.7, 135.52, 135.47, 132.6, 129.4, 129.1, 128.4, 128.1, 127.6, 126.6, 125.3, 124.2, 123.9, 122.8, 120.8, 119.9, 119.0, $97.6,68.8,61.9,55.1,40.3,31.9,30.6,30.1,29.34,29.31,25.6,24.0,22.7,19.4,14.2,-0.6,-0.7$; IR (KBr): 3458, 2926, 2853, 1591, 1514, 1493, 1464, 1281, 1028, 837, 816, 752, $696 \mathrm{~cm}^{-1}$; Anal. Calcd for $\mathrm{C}_{61} \mathrm{H}_{75} \mathrm{NO}_{2} \mathrm{Si}: \mathrm{C}, 83.04 ; \mathrm{H}, 8.57$. Found: $\mathrm{C}, 83.14 ; \mathrm{H}, 8.55$.

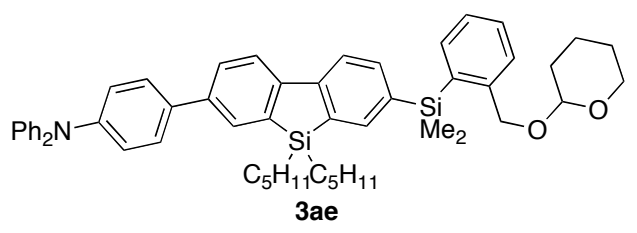

7-[4-(Diphenylamino)phenyl]-2-([2-(tetrahydro-2H-pyranoxymethyl)phenyl]dimethylsilyl)-9,9'-dipentyl-9H-9-silafluo rene (3ae). A pale yellow viscous liquid, $\mathrm{R}_{\mathrm{f}} 0.27$ (hexane-ethyl acetate $=10: 1) .{ }^{1} \mathrm{H} \mathrm{NMR}\left(400 \mathrm{MHz}, \mathrm{CDCl}_{3}\right) \delta 7.84(\mathrm{~d}, J=8.1$ $\mathrm{Hz}, 1 \mathrm{H}), 7.81-7.71(\mathrm{~m}, 1 \mathrm{H}), 7.61(\mathrm{dd}, J=8.1,1.3 \mathrm{~Hz}, 1 \mathrm{H})$, 7.57-7.46 (m, 1H), $7.39(\mathrm{t}, J=7.4 \mathrm{~Hz}, 1 \mathrm{H}), 7.31-7.21(\mathrm{~m}, 5 \mathrm{H}), 7.18-7.09(\mathrm{~m}, 6 \mathrm{H}), 7.02(\mathrm{t}, J=7.2 \mathrm{~Hz}$, 
2H), $4.69(\mathrm{~d}, J=12.1 \mathrm{~Hz}, 1 \mathrm{H}), 4.48(\mathrm{t}, J=3.4 \mathrm{~Hz}, 1 \mathrm{H}), 4.43(\mathrm{~d}, J=11.9 \mathrm{~Hz}, 1 \mathrm{H}), 3.82-3.71(\mathrm{~m}, 1 \mathrm{H})$, 3.46-3.36 (m, 1H), 1.85-1.72 (m, 1H), 1.67-1.16 (m, 17H), 0.98-0.89 (m, 4H), 0.85-0.75 (m, 6H), $0.630(\mathrm{~s}, 3 \mathrm{H}), 0.626(\mathrm{~s}, 3 \mathrm{H}) ;{ }^{13} \mathrm{C} \mathrm{NMR}\left(101 \mathrm{MHz}, \mathrm{CDCl}_{3}\right) \delta 148.9,147.8,147.2,147.1,144.3,139.6$, 139.01, 139.03, 137.33, 137.30, 136.6, 136.2, 135.8, 135.4, 131.5, 129.7, 129.4, 128.7, 128.6, 127.8, 127.0, 124.5, 124.2, 123.1, 121.4, 120.4, 98.0, 69.1, 62.3, 35.9, 30.9, 25.9, 24.0, 22.6, 19.7, 14.4, 12.7, $-0.3,-0.4$; IR (KBr): 3450, 2953, 2922, 2870, 2855, 1591, 1510, 1493, 1452, 1325, 1279, 1117, 1076, 1026, 837, 816, 752, 696, $498 \mathrm{~cm}^{-1}$; Anal. Calcd for $\mathrm{C}_{54} \mathrm{H}_{63} \mathrm{NO}_{2} \mathrm{Si}_{2}: \mathrm{C}$, 79.65; H, 7.80. Found: C, 79.65; H, 7.80 .

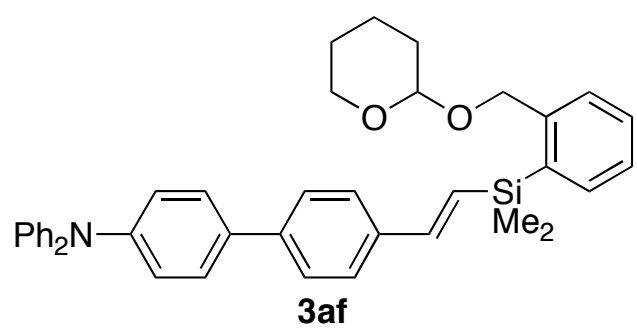

(E)-4-(Diphenylamino)-4'-[2-([2-(tetrahydro-2H-pyranoxymethyl)phenyl]dimethylsilyl)ethenyl]biphenyl (3af). A yellow solid, $\mathrm{mp} 47.0-49.2^{\circ} \mathrm{C}, \mathrm{R}_{\mathrm{f}} 0.21$ (hexane-ethyl acetate $=$ 10:1). ${ }^{1} \mathrm{H}$ NMR $\left(400 \mathrm{MHz}, \mathrm{CDCl}_{3}\right) \delta 7.60(\mathrm{~d}, J=7.3 \mathrm{~Hz}, 1 \mathrm{H})$, $7.57-7.44(\mathrm{~m}, 7 \mathrm{H}), 7.40(\mathrm{td}, J=7.5,1.4 \mathrm{~Hz}, 1 \mathrm{H}), 7.33-7.22(\mathrm{~m}$, $5 \mathrm{H}), 7.16-7.08(\mathrm{~m}, 6 \mathrm{H}), 7.03(\mathrm{t}, J=7.3 \mathrm{~Hz}, 2 \mathrm{H}), 6.96(\mathrm{~d}, J$ $=19.0 \mathrm{~Hz}, 1 \mathrm{H}), 6.68(\mathrm{~d}, J=19.0 \mathrm{~Hz}, 1 \mathrm{H}), 4.88(\mathrm{~d}, J=11.9 \mathrm{~Hz}$, $1 \mathrm{H}), 4.67(\mathrm{t}, J=3.5 \mathrm{~Hz}, 1 \mathrm{H}), 4.63(\mathrm{~d}, J=11.9 \mathrm{~Hz}, 1 \mathrm{H})$,

3.94-3.84 (m, 1H), 3.53-3.43 (m, 1H), 1.90-1.78 (m, 1H) 1.75-1.43 (m, 5H), 0.51 (s, 6H); ${ }^{13} \mathrm{C}$ NMR $\left(101 \mathrm{MHz}, \mathrm{CDCl}_{3}\right) \delta 147.4,147.1,144.2,143.8,140.1,136.63,136.60,134.9,134.3,129.4,129.1$, $128.4,127.5,127.4,126.81,126.76,126.5,124.3,123.7,122.8,97.9,68.9,62.2,30.7,25.6,19.5,-1.0$, -1.1; IR (KBr): 3055, 3032, 2943, 2868, 1589, 1489, 1325, 1277, 1200, 1180, 1117, 1076, 1024, 988, 905, 818, 752, $694 \mathrm{~cm}^{-1}$; HRMS (EI) Calcd for $\mathrm{C}_{40} \mathrm{H}_{41} \mathrm{NO}_{2} \mathrm{Si}: \mathrm{M}^{+}, 595.2907$. Found: $m / z$ 595.2902.

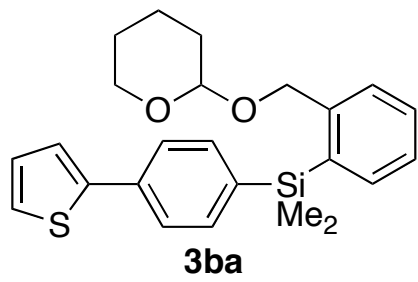

Dimethyl[2-(tetrahydro-2H-pyranoxymethyl)phenyl][4-(2-thienyl)phenyl]silane (3ba). A pale yellow viscous oil, $\mathrm{R}_{\mathrm{f}} 0.13$ (hexane-ethyl acetate $=$ 20:1). ${ }^{1} \mathrm{H}$ NMR $\left(400 \mathrm{MHz}, \mathrm{CDCl}_{3}\right) \delta 7.60-7.46(\mathrm{~m}, 6 \mathrm{H}), 7.42$ (td, $J=7.5$, $1.5 \mathrm{~Hz}, 1 \mathrm{H}), 7.34-7.24(\mathrm{~m}, 3 \mathrm{H}), 7.08(\mathrm{dd}, J=5.0,3.6 \mathrm{~Hz}, 1 \mathrm{H}), 4.68(\mathrm{~d}, J=$ $12.1 \mathrm{~Hz}, 1 \mathrm{H}), 4.45(\mathrm{t}, J=3.6 \mathrm{~Hz}, 1 \mathrm{H}), 4.40(\mathrm{~d}, J=11.9 \mathrm{~Hz}, 1 \mathrm{H}), 3.79-3.71$

$(\mathrm{m}, 1 \mathrm{H}), 3.45-3.37(\mathrm{~m}, 1 \mathrm{H}), 1.84-1.72(\mathrm{~m}, 1 \mathrm{H}), 1.67-1.39(\mathrm{~m}, 5 \mathrm{H}), 0.620(\mathrm{~s}$, 3H), $0.615(\mathrm{~s}, 3 \mathrm{H}) ;{ }^{13} \mathrm{C}$ NMR $\left(101 \mathrm{MHz}, \mathrm{CDCl}_{3}\right) \delta 144.2,144.0,138.0,135.8,135.3,134.7,134.5$, 129.6, 128.5, 127.9, 126.7, 125.1, 124.8, 123.1, 97.8, 68.8, 62.0, 30.6, 25.5, 19.4, -0.8, -0.9; IR (neat): 3055, 3013, 2945, 2870, 1597, 1433, 1394, 1350, 1258, 1200, 1117, 1078, 1026, 974, 907, 870, 833, 812, 775, 756, 729, 696, 654, $534 \mathrm{~cm}^{-1}$; Anal. Calcd for $\mathrm{C}_{24} \mathrm{H}_{28} \mathrm{O}_{2} \mathrm{SSi}_{2}: \mathrm{C}, 70.54 ; \mathrm{H}, 6.91$. Found: C, $70.46 ; \mathrm{H}, 6.84$

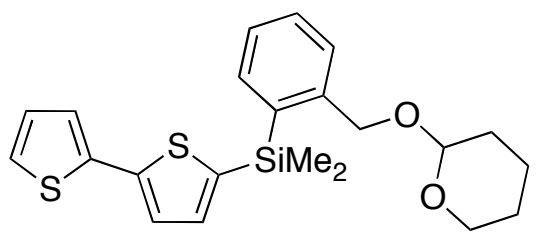

$3 b b$

5-([2-(tetrahydro-2H-pyranoxymethyl)phenyl]dimethylsilyl)-2,2'bithiophene (3bb). To a mixture of $\mathbf{1 b}(6.0 \mathrm{~g}, 24 \mathrm{mmol}), \mathrm{K}_{2} \mathrm{CO}_{3}(6.9$ $\mathrm{g}, 50 \mathrm{mmol})$, (dppf) $\mathrm{PdCl}_{2} \bullet \mathrm{CH}_{2} \mathrm{Cl}_{2}(163 \mathrm{mg}, 0.20 \mathrm{mmol})$, and $\mathrm{CuI}$ (114 $\mathrm{mg}, 0.60 \mathrm{mmol})$ in DMF $(16 \mathrm{~mL})$ and THF $(44 \mathrm{~mL})$ in a Schlenk tube was added $\mathbf{2 b}(20 \mathrm{mmol})$, and the resulting mixture was stirred at $75^{\circ} \mathrm{C}$ for $6 \mathrm{~h}$. The mixture was filtered through a Florisil pad, diluted with $\mathrm{Et}_{2} \mathrm{O}$, washed with water and brine, dried over anhydrous $\mathrm{MgSO}_{4}$, and then concentrated in vacuo. The residue was purified by flash chromatography on silica gel to afford $3 \mathbf{b b}(8.0 \mathrm{~g}, 96 \%)$ as a colorless viscous oil, $\mathrm{R}_{\mathrm{f}} 0.20$ (hexane-ethyl acetate $=15: 1$ ). ${ }^{1} \mathrm{H}$ NMR $\left(400 \mathrm{MHz}, \mathrm{CDCl}_{3}\right) \delta 7.54(\mathrm{dd}, J=7.3,1.3 \mathrm{~Hz}, 1 \mathrm{H}), 7.49(\mathrm{~d}, J=7.7 \mathrm{~Hz}, 1 \mathrm{H}), 7.41(\mathrm{td}, J=7.5$, $1.3 \mathrm{~Hz}, 1 \mathrm{H}), 7.28(\mathrm{td}, J=7.3,1.1 \mathrm{~Hz}, 1 \mathrm{H}), 7.21(\mathrm{~d}, J=3.5 \mathrm{~Hz}, 1 \mathrm{H}), 7.19(\mathrm{dd}, J=5.1,1.1 \mathrm{~Hz}, 1 \mathrm{H}), 7.15$ $(\mathrm{dd}, J=3.7,1.1 \mathrm{~Hz}, 1 \mathrm{H}), 7.12(\mathrm{~d}, J=3.5 \mathrm{~Hz}, 1 \mathrm{H}), 6.99(\mathrm{dd}, J=5.0,3.6 \mathrm{~Hz}, 1 \mathrm{H}), 4.77(\mathrm{~d}, J=11.9 \mathrm{~Hz}$, $1 \mathrm{H}), 4.56(\mathrm{t}, J=3.6 \mathrm{~Hz}, 1 \mathrm{H}), 4.51(\mathrm{~d}, J=11.9 \mathrm{~Hz}, 1 \mathrm{H}), 3.86-3.77(\mathrm{~m}, 1 \mathrm{H}), 3.51-3.41(\mathrm{~m}, 1 \mathrm{H})$, 
$1.88-1.75(\mathrm{~m}, 1 \mathrm{H}), 1.72-1.41(\mathrm{~m}, 5 \mathrm{H}), 0.66(\mathrm{~s}, 6 \mathrm{H}) ;{ }^{13} \mathrm{C} \mathrm{NMR}\left(101 \mathrm{MHz}, \mathrm{CDCl}_{3}\right) \delta$ 143.9, 142.9, 137.9, 137.1, 135.9, 135.4, 135.2, 129.8, 128.6, 127.7, 126.8, 124.9, 124.3, 123.7, 97.8, 68.8, 62.1, 30.6, 25.6, 19.5, 0.3, 0.2; IR (neat): 2943, 2870, 2849, 1439, 1252, 1200, 1119, 1078, 1026, 988, 907, 837, 812, 777, 756, $692 \mathrm{~cm}^{-1}$; Anal. Calcd for $\mathrm{C}_{22} \mathrm{H}_{26} \mathrm{O}_{2} \mathrm{~S}_{2} \mathrm{Si}$ : C, 63.72; H, 6.32. Found: C, 63.47; H, 6.32.

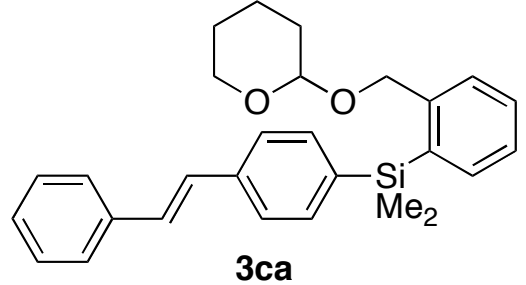

(E)-4-(Dimethyl[2-(tetrahydro-2H-pyranoxymethyl)phenyl]silyl)stilbene (3ca). A pale yellow viscous oil, $\mathrm{R}_{\mathrm{f}} 0.10$ (hexane-ethyl acetate $=20: 1) .{ }^{1} \mathrm{H}$ NMR $\left(400 \mathrm{MHz}, \mathrm{CDCl}_{3}\right) \delta$ 7.57-7.45 (m, 8H), $7.42(\mathrm{td}, J=7.6,1.4 \mathrm{~Hz}, 1 \mathrm{H}), 7.39-7.33(\mathrm{~m}, 2 \mathrm{H}), 7.32-7.23(\mathrm{~m}, 2 \mathrm{H})$, $7.14(\mathrm{~d}, J=16.3,1 \mathrm{H}), 7.09(\mathrm{~d}, J=16.3 \mathrm{~Hz}, 1 \mathrm{H}), 4.68(\mathrm{~d}, J=12.1 \mathrm{~Hz}$, $1 \mathrm{H}), 4.59(\mathrm{t}, J=3.5,1 \mathrm{H}), 4.40(\mathrm{~d}, J=11.9 \mathrm{~Hz}, 1 \mathrm{H}), 3.80-3.71(\mathrm{~m}$, $1 \mathrm{H}), 3.46-3.37(\mathrm{~m}, 1 \mathrm{H}), 1.82-1.73(\mathrm{~m}, 1 \mathrm{H}), 1.69-1.41(\mathrm{~m}, 5 \mathrm{H}), 0.62$

(s, 3H), $0.61(\mathrm{~s}, 3 \mathrm{H}) ;{ }^{13} \mathrm{C}$ NMR $\left(101 \mathrm{MHz}, \mathrm{CDCl}_{3}\right) \delta 144.0,138.2,137.7,137.1,135.9,135.3,134.3$, $129.5,128.9,128.54,128.48,128.4,127.5,126.7,126.4,125.7,97.8,68.8,62.0,30.6,25.5,19.4,-0.8$, -0.9; IR (neat) 3057, 3024, 2949, 2870, 1597, 1495, 1466, 1448, 1439, 1396, 1348, 1258, 1200, 1117 , 1078, 1026, 964, 907, 870, 835, 806, 775, 754, 718, 692, 646, 577, $536 \mathrm{~cm}^{-1}$; Anal. Calcd for $\mathrm{C}_{28} \mathrm{H}_{32} \mathrm{O}_{2} \mathrm{Si}: \mathrm{C}, 78.46 ; \mathrm{H}, 7.52$. Found: C, 78.40; H, 7.59

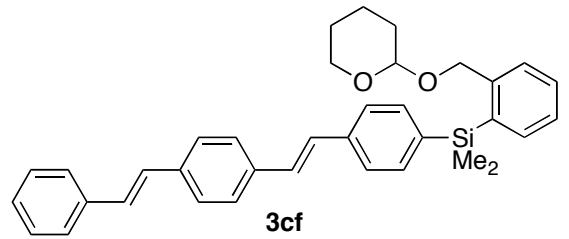

(E)-1-(2-[4-(Dimethyl[2-(tetrahydro-2H-pyranoxymethyl)phenyl ]silyl)phenyl]ethenyl)-4-(2-phenyl)ethenylbenzene (3cf). A yellow solid, $\mathrm{mp} 53.2-56.5{ }^{\circ} \mathrm{C}, \mathrm{R}_{\mathrm{f}} 0.13$ (hexane-ethyl acetate $=$ 20:1). ${ }^{1} \mathrm{H} \mathrm{NMR}\left(400 \mathrm{MHz}, \mathrm{CDCl}_{3}\right) \delta 7.59(\mathrm{dd}, J=7.4,1.2 \mathrm{~Hz}, 1 \mathrm{H})$, 7.56-7.22 (m, 8H), $7.13(\mathrm{~d}, J=16.3 \mathrm{~Hz}, 1 \mathrm{H}), 7.08(\mathrm{~d}, J=16.3 \mathrm{~Hz}$, $1 \mathrm{H}), 6.93(\mathrm{~d}, J=19.2 \mathrm{~Hz}, 1 \mathrm{H}), 6.67(\mathrm{~d}, J=19.0 \mathrm{~Hz}, 1 \mathrm{H}), 4.87(\mathrm{~d}, J$ $=11.9 \mathrm{~Hz}, 1 \mathrm{H}), 4.67(\mathrm{t}, J=3.5 \mathrm{~Hz}, 1 \mathrm{H}), 4.62(\mathrm{~d}, J=11.9 \mathrm{~Hz}, 1 \mathrm{H}), 3.93-3.84(\mathrm{~m}, 1 \mathrm{H}), 3.52-3.44(\mathrm{~m}, 1 \mathrm{H})$, $1.92-1.78(\mathrm{~m}, 1 \mathrm{H}), 1.76-1.43(\mathrm{~m}, 5 \mathrm{H}), 0.51(\mathrm{~s}, 6 \mathrm{H}) ;{ }^{13} \mathrm{C} \mathrm{NMR}\left(101 \mathrm{MHz}, \mathrm{CDCl}_{3}\right) \delta$ 144.2, 143.8, 137.4, $137.1,137.0,136.6,134.9,129.4,128.6,128.4,128.1,127.7,127.5,126.8,126.7,126.5,126.4,97.9$, 68.9, 62.2, 30.7, 25.6, 19.5, -1.0, -1.1; IR (KBr): 3053, 3022, 2941, 2891, 2868, 1597, 1508, 1448, 1437, 1342, 1250, 1200, 1117, 1076, 1055, 1028, 991, 966, 907, 868, 837, 820, 793, 752, 731, 708, 689, $534 \mathrm{~cm}^{-1}$; Anal. Calcd for $\mathrm{C}_{30} \mathrm{H}_{34} \mathrm{O}_{2} \mathrm{Si}_{2}$ : C, 79.25; H, 7.54. Found: C, 79.10; H, 7.46.

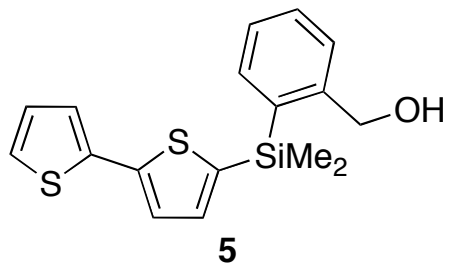

Deprotection of 3bb. A solution of $3 \mathbf{b b}(3.4 \mathrm{~g}, 8.3 \mathrm{mmol})$ and PPTS (0.42 $\mathrm{g}, 1.65 \mathrm{mmol})$ in $\mathrm{MeOH}(41 \mathrm{~mL})$ was stirred at $40{ }^{\circ} \mathrm{C}$ for $2 \mathrm{~h}$, and then concentrated in vacuo. The resulting residue was purified by flash chromatography on silica gel to give 5-([2-(hydroxymethyl)phenyl]dimethylsilyl)-2,2'-bithiophene $(5,2.4 \mathrm{~g}$, $89 \%$ ) as a white solid, $\mathrm{mp} 47.2-48.0{ }^{\circ} \mathrm{C}, \mathrm{R}_{\mathrm{f}} 0.30$ (hexane-ethyl acetate $=$ 5:1). ${ }^{1} \mathrm{H}$ NMR $\left(400 \mathrm{MHz}, \mathrm{CDCl}_{3}\right) \delta 7.57(\mathrm{~d}, J=7.3 \mathrm{~Hz}, 1 \mathrm{H}), 7.50-7.39(\mathrm{~m}, 2 \mathrm{H}), 7.30(\mathrm{t}, J=7.2 \mathrm{~Hz}$, $1 \mathrm{H}), 7.21(\mathrm{~d}, J=3.5 \mathrm{~Hz}, 1 \mathrm{H}), 7.19(\mathrm{~d}, J=5.1 \mathrm{~Hz}, 1 \mathrm{H}), 7.15(\mathrm{~d}, J=3.5 \mathrm{~Hz}, 1 \mathrm{H}), 7.13(\mathrm{~d}, J=3.5 \mathrm{~Hz}$, $1 \mathrm{H}), 6.98(\mathrm{dd}, J=4.8,3.8 \mathrm{~Hz}, 1 \mathrm{H}), 4.67(\mathrm{~d}, J=5.5 \mathrm{~Hz}, 2 \mathrm{H}), 1.53(\mathrm{t}, J=5.8 \mathrm{~Hz}, 1 \mathrm{H}), 0.67(\mathrm{~s}, 6 \mathrm{H}) ;{ }^{13} \mathrm{C}$ NMR $\left(101 \mathrm{MHz}, \mathrm{CDCl}_{3}\right) \delta 146.4,143.1,137.8,136.9,135.9,135.2,135.0,130.1,128.0,127.7,127.0$, 125.0, 124.5, 123.9, 65.3, 0.3; IR (KBr): 3329, 3057, 2947, 1439, 1418, 1217, 1200, 1126, 1078, 1015, 989, 839, 810, 779, 758, 746, 708, $691 \mathrm{~cm}^{-1}$; Anal. Calcd for $\mathrm{C}_{17} \mathrm{H}_{18} \mathrm{OS}_{2} \mathrm{Si}: \mathrm{C}, 61.77 ; \mathrm{H}, 5.49$. Found: C, $61.58 ; \mathrm{H}, 5.48$. 


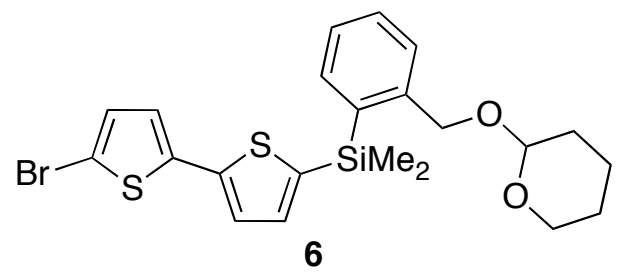

Bromination of 3bb. To a solution of $3 \mathbf{b b}(4.6 \mathrm{~g}, 11 \mathrm{mmol})$ in $\mathrm{Et}_{2} \mathrm{O}(33 \mathrm{~mL})$ was added TMEDA $(1.41 \mathrm{~g}, 12.1 \mathrm{mmol})$ and a 1.6 M solution of $n$-BuLi $(7.2 \mathrm{~mL}, 11.6 \mathrm{mmol})$ in hexane at $-40{ }^{\circ} \mathrm{C}$, and the resulting mixture was stirred at $-40{ }^{\circ} \mathrm{C}$ for $30 \mathrm{~min}$ and then at $\mathrm{rt}$ for $30 \mathrm{~min}$. To this was added 1,2-dibromo-1,1,2,2-tetrafluoroethane $(3.1 \mathrm{~g}, 12.1 \mathrm{mmol})$ at $-40{ }^{\circ} \mathrm{C}$, and the resulting mixture was stirred at the same temperature for $1 \mathrm{~h}$. The reaction was quenched with a saturated $\mathrm{Na}_{2} \mathrm{~S}_{2} \mathrm{O}_{3}$ aqueous solution $(20 \mathrm{~mL})$, and the aqueous layer was extracted with diethyl ether $(120 \mathrm{~mL})$. The organic layers were dried over anhydrous $\mathrm{MgSO}_{4}$, concentrated in vacuo, and then purified by flash chromatography on silica gel to give 5-bromo-5'-(dimethyl[2-(tetrahydro-2H-pyranoxymethyl)phenyl] silyl)-2,2'-bithiophene $\quad(6, \quad 4.9 \quad \mathrm{~g}$, $90 \%)$ as a colorless oil, $\mathrm{R}_{\mathrm{f}} 0.22$ (hexane-ethyl acetate $\left.=15: 1\right) .{ }^{1} \mathrm{H}$ NMR $\left(400 \mathrm{MHz}, \mathrm{CDCl}_{3}\right) \delta 7.54(\mathrm{~d}, J$ $=7.3 \mathrm{~Hz}, 1 \mathrm{H}), 7.50(\mathrm{~d}, J=7.5 \mathrm{~Hz}, 1 \mathrm{H}), 7.42(\mathrm{td}, J=7.4,1.0 \mathrm{~Hz}, 1 \mathrm{H}), 7.29(\mathrm{t}, J=7.3 \mathrm{~Hz}, 1 \mathrm{H}), 7.15(\mathrm{~d}$, $J=3.5 \mathrm{~Hz}, 1 \mathrm{H}), 7.12(\mathrm{~d}, J=3.5 \mathrm{~Hz}, 1 \mathrm{H}), 6.95(\mathrm{~d}, J=3.8 \mathrm{~Hz}, 1 \mathrm{H}), 6.90(\mathrm{~d}, J=3.8 \mathrm{~Hz}, 1 \mathrm{H}), 4.77(\mathrm{~d}, J$ $=12.1 \mathrm{~Hz}, 1 \mathrm{H}), 4.56(\mathrm{t}, J=3.5 \mathrm{~Hz}, 1 \mathrm{H}), 4.50(\mathrm{~d}, J=12.1 \mathrm{~Hz}, 1 \mathrm{H}), 3.88-3.76(\mathrm{~m}, 1 \mathrm{H}), 3.52-3.42(\mathrm{~m}$, $1 \mathrm{H}), 1.73-1.42(\mathrm{~m}, 5 \mathrm{H}), 1.88-17.5(\mathrm{~m}, 1 \mathrm{H}), 0.67(\mathrm{~s}, 6 \mathrm{H}) ;{ }^{13} \mathrm{C}$ NMR $\left(101 \mathrm{MHz}, \mathrm{CDCl}_{3}\right) \delta 143.9,141.8$, 138.63, 138.60, 135.9, 135.22, 135.17, 130.5, 129.9, 128.6, 126.8, 125.1, 123.8, 110.9, 97.8, 68.7, 62.1, 30.6, 25.5, 19.5, 0.3, 0.2 ; IR (neat): 3055, 2943, 2870, 2849, 1441, 1418, 1350, 1252, 1200, 1119, 1078, 1026, 988, 970, 905, 870, 835, 812, 754, 691, 654, $530 \mathrm{~cm}^{-1}$; Anal. Calcd for $\mathrm{C}_{22} \mathrm{H}_{25} \mathrm{BrO}_{2} \mathrm{~S}_{2} \mathrm{Si}: \mathrm{C}$, 53.54; H, 5.11. Found: C, 53.77; H, 5.19.

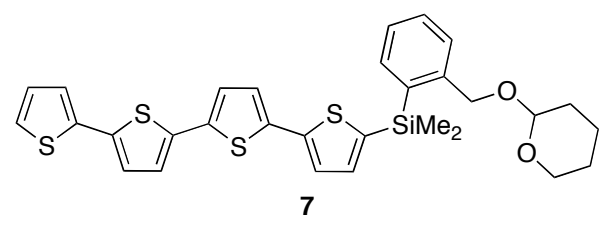

Synthesis of 7. To a solution of $5(3.9 \mathrm{~g}, 11.9 \mathrm{mmol}), \mathrm{K}_{2} \mathrm{CO}_{3}(3.4$ $\mathrm{g}, 25 \mathrm{mmol})$, (dppf) $\mathrm{PdCl}_{2} \bullet \mathrm{CH}_{2} \mathrm{Cl}_{2}(0.25 \mathrm{~g}, 0.30 \mathrm{mmol})$, and $\mathrm{CuI}$ $(0.17 \mathrm{~g}, 0.89 \mathrm{mmol})$ in DMF $(7.9 \mathrm{~mL})$ and THF $(22 \mathrm{~mL})$ in a Schlenk tube was added $6(4.9 \mathrm{~g}, 9.9 \mathrm{mmol})$, and the resulting mixture was stirred at $75{ }^{\circ} \mathrm{C}$ for $10 \mathrm{~h}$ before filtration through a Frolisile pad. After concentration in vacuo, the residue was filtered through a short silica gel column and further purified by preparative GPC to afford 5-([2-(tetrahydro-2H-pyranoxymethyl)phenyl]dimethylsilyl)-2,2':5',2', 5', ,2',' -quarterthiophene (7, $4.7 \mathrm{~g}, 82 \%)$ as a yellow solid, $\mathrm{mp} 111.0-111.7{ }^{\circ} \mathrm{C} .{ }^{1} \mathrm{H}$ NMR $\left(400 \mathrm{MHz}, \mathrm{CDCl}_{3}\right) \delta 7.55(\mathrm{dd}, J=7.5,1.2$ $\mathrm{Hz}, 1 \mathrm{H}), 7.50(\mathrm{~d}, J=7.6 \mathrm{~Hz}, 1 \mathrm{H}), 7.42(\mathrm{td}, J=7.5,1.3 \mathrm{~Hz}, 1 \mathrm{H}), 7.29(\mathrm{td}, J=7.3,1.3 \mathrm{~Hz}, 1 \mathrm{H})$, $7.23-7.18(\mathrm{~m}, 2 \mathrm{H}), 7.16(\mathrm{dd}, J=3.7,1.1 \mathrm{~Hz}, 1 \mathrm{H}), 7.13(\mathrm{~d}, J=3.5 \mathrm{~Hz}, 1 \mathrm{H}), 7.09-7.03(\mathrm{~m}, 4 \mathrm{H}), 7.02(\mathrm{dd}$, $J=5.1,3.7 \mathrm{~Hz}, 1 \mathrm{H}), 4.77(\mathrm{~d}, J=11.9 \mathrm{~Hz}, 1 \mathrm{H}), 4.56(\mathrm{t}, J=3.6 \mathrm{~Hz}, 1 \mathrm{H}), 4.51(\mathrm{~d}, J=11.9 \mathrm{~Hz}, 1 \mathrm{H})$, 3.85-3.77 (m, 1H), 3.51-3.42 (m, 1H), 1.87-1.75 (m, 1H), 1.72-1.42 (m, 5H), $0.67(\mathrm{~s}, 6 \mathrm{H})$; ${ }^{13} \mathrm{C}$ NMR $\left(101 \mathrm{MHz}, \mathrm{CDCl}_{3}\right) \delta 143.9,142.5,138.3,136.9,136.1,136.03,136.01,135.8,135.7,135.3,135.2$, 129.8, 128.6, 127.8, 126.8, 124.9, 124.43, 124.40, 124.3, 124.2, 124.1, 123.6, 97.8, 68.8, 62.1, 30.6, 25.6, 19.5, 0.3, 0.2; IR (KBr): 3452, 2949, 833, 793, $687 \mathrm{~cm}^{-1}$; Anal. Calcd for $\mathrm{C}_{30} \mathrm{H}_{30} \mathrm{O}_{2} \mathrm{~S}_{4} \mathrm{Si}: \mathrm{C}, 62.24$; H, 5.22. Found: C, 62.00; H, 4.95.

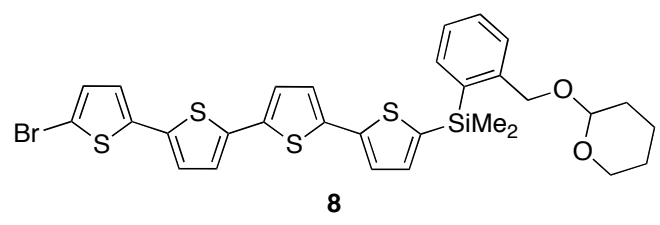

Bromination of 7. To a solution of $7(0.70 \mathrm{~g}, 1.2 \mathrm{mmol})$ in THF (14 mL) were added TMEDA (153 mg, $1.32 \mathrm{mmol})$ and a $1.6 \mathrm{M}$ solution of $n$-BuLi $(1.32 \mathrm{mmol})$ in hexane at $-78{ }^{\circ} \mathrm{C}$, and the resulting mixture was stirred at the same temperature for $5 \mathrm{~min}$. To this was added $\mathrm{BrCF}_{2} \mathrm{CF}_{2} \mathrm{Br}(0.37 \mathrm{~g}, 1.44 \mathrm{mmol})$, and the resulting mixture was stirred at $-40{ }^{\circ} \mathrm{C}$ for $1 \mathrm{~h}$ and then quenched with a saturated $\mathrm{Na}_{2} \mathrm{~S}_{2} \mathrm{O}_{3}$ aqueous solution $(2 \mathrm{~mL})$. The aqueous layer was extracted with diethyl ether $(20 \mathrm{~mL})$, and the organic layers were dried over anhydrous $\mathrm{MgSO}_{4}$ and concentrated in vacuo. The residue was purified by preparative GPC 
ja074728s_SI_0824.pdf

5-bromo-5', '-(dimethyl[2-(tetrahydro-2H-pyranoxymethyl)phenyl]silyl)-2,2':5',2' :5', ,2','-quarterthio phene $(8,0.71 \mathrm{~g}, 90 \%)$ as a yellow solid, mp 112.4-115.7 ${ }^{\circ} \mathrm{C} .{ }^{1} \mathrm{H}$ NMR $\left(400 \mathrm{MHz}, \mathrm{CDCl}_{3}\right) \delta 7.54(\mathrm{dd}$, $J=7.3,1.1 \mathrm{~Hz}, 1 \mathrm{H}), 7.50(\mathrm{~d}, J=8.1 \mathrm{~Hz}, 1 \mathrm{H}), 7.41(\mathrm{td}, J=7.5,1.3 \mathrm{~Hz}, 1 \mathrm{H}), 7.29(\mathrm{td}, J=7.4,1.0 \mathrm{~Hz}$, $1 \mathrm{H}), 7.21(\mathrm{~d}, J=3.5 \mathrm{~Hz}, 1 \mathrm{H}), 7.13(\mathrm{~d}, J=3.5 \mathrm{~Hz}, 1 \mathrm{H}), 7.08-7.02(\mathrm{~m}, 3 \mathrm{H}), 7.00(\mathrm{~d}, J=3.7 \mathrm{~Hz}, 1 \mathrm{H})$, $6.97(\mathrm{~d}, J=3.8 \mathrm{~Hz}, 1 \mathrm{H}), 6.90(\mathrm{~d}, J=3.8 \mathrm{~Hz}, 1 \mathrm{H}), 4.77(\mathrm{~d}, J=12.1 \mathrm{~Hz}, 1 \mathrm{H}), 4.56(\mathrm{t}, J=3.5 \mathrm{~Hz}, 1 \mathrm{H})$, $4.51(\mathrm{~d}, J=11.9 \mathrm{~Hz}, 1 \mathrm{H}), 3.86-3.77(\mathrm{~m}, 1 \mathrm{H}), 3.51-3.43(\mathrm{~m}, 1 \mathrm{H}), 1.86-1.74(\mathrm{~m}, 1 \mathrm{H}), 1.72-1.42(\mathrm{~m}$, 5H), $0.67(\mathrm{~s}, 6 \mathrm{H}) ;{ }^{13} \mathrm{C}$ NMR $\left(101 \mathrm{MHz}, \mathrm{CDCl}_{3}\right) \delta 143.9,142.3,138.4,138.3,136.3,136.2,136.0,135.4$, $135.3,135.2$, 134.9, 130.5, 129.8, 128.6, 126.8, 124.9, 124.5, 124.38, 124.37, 124.0, 123.6, 111.0, 97.8, 68.7, 62.1, 30.6, 25.5, 19.5, 0.3, 0.2; IR (KBr): 3450, 3061, 2943, 2870, 1427, 1254, 1117, 1078, 1028, 989, 843, 835, 814, 789, 756, $465 \mathrm{~cm}^{-1}$; HRMS (FAB+) Calcd for $\mathrm{C}_{30} \mathrm{H}_{29} \mathrm{BrO}_{2} \mathrm{~S}_{4} \mathrm{Si}: \mathrm{M}^{+}, 656.0003$. Found: $m / z 656.0024$.

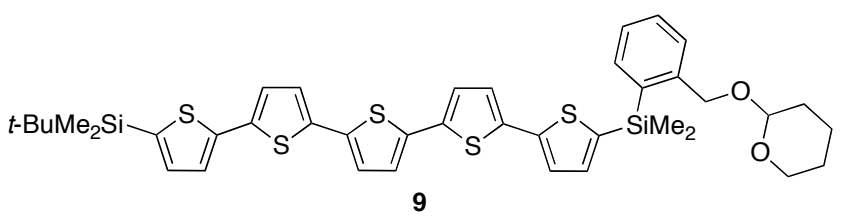

Synthesis of 9. To a solution of $\mathbf{1 d}(44 \mathrm{mg}, 0.12$ $\mathrm{mmol}), \quad \mathrm{K}_{2} \mathrm{CO}_{3} \quad(3.5 \quad \mathrm{mg}, \quad 0.25 \quad \mathrm{mmol})$, (dppf) $\mathrm{PdCl}_{2} \bullet \mathrm{CH}_{2} \mathrm{Cl}_{2}(4.1 \mathrm{mg}, 5.0 \mu \mathrm{mol})$, and $\mathrm{CuI}$ $(1.0 \mathrm{mg}, 5.0 \mu \mathrm{mol})$ in $\mathrm{DMF}(0.16 \mathrm{~mL})$ and THF $(0.44 \mathrm{~mL})$ in a Schlenk tube was added $8(66 \mathrm{mg}$, $0.10 \mathrm{mmol}$ ), and the resulting mixture was stirred at $50{ }^{\circ} \mathrm{C}$ for $5 \mathrm{~h}$ before filtration through a Florisil pad. After concentration in vacuo, the residue was filtered through a short silica gel column and further purified by preparative GPC to give 5',' '-(tert-butyldimethylsilyl)-5-(dimethyl[2-(tetrahydro-2H-pyranoxymethyl)phenyl]silyl)-2,2':5',2', 5 ',2" ':5', ,2",' -quinquethiophene $(9,62 \mathrm{mg}, 80 \%)$ as an orange solid, mp 128.5-130.3 ${ }^{\circ} \mathrm{C}, \mathrm{R}_{\mathrm{f}} 0.32$ (hexane-ethyl acetate = 5:1). ${ }^{1} \mathrm{H}$ NMR $\left(400 \mathrm{MHz} \mathrm{CDCl}_{3}\right) \delta 7.55(\mathrm{~d}, J=7.3 \mathrm{~Hz}, 1 \mathrm{H}), 7.50(\mathrm{~d}, J=7.5$ $\mathrm{Hz}, 1 \mathrm{H}), 7.42(\mathrm{t}, J=7.5 \mathrm{~Hz}, 1 \mathrm{H}), 7.29(\mathrm{t}, J=7.3 \mathrm{~Hz}, 1 \mathrm{H}), 7.23(\mathrm{~d}, J=3.3 \mathrm{~Hz}, 1 \mathrm{H}), 7.21(\mathrm{~d}, J=3.5 \mathrm{~Hz}$, $1 \mathrm{H}), 7.13(\mathrm{~d}, J=3.5 \mathrm{~Hz}, 1 \mathrm{H}), 7.11-7.01(\mathrm{~m}, 7 \mathrm{H}), 4.77(\mathrm{~d}, J=11.9 \mathrm{~Hz}, 1 \mathrm{H}), 4.56(\mathrm{t}, J=3.4 \mathrm{~Hz}, 1 \mathrm{H})$, $4.51(\mathrm{~d}, J=11.9 \mathrm{~Hz}, 1 \mathrm{H}), 3.87-3.77(\mathrm{~m}, 1 \mathrm{H}), 3.51-3.42(\mathrm{~m}, 1 \mathrm{H}), 1.88-1.75(\mathrm{~m}, 1 \mathrm{H}), 1.73-1.43(\mathrm{~m}$, $5 \mathrm{H}), 0.95(\mathrm{~s}, 9 \mathrm{H}), 0.67(\mathrm{~s}, 6 \mathrm{H}), 0.31(\mathrm{~s}, 6 \mathrm{H}) ;{ }^{13} \mathrm{C} \mathrm{NMR}\left(101 \mathrm{MHz}, \mathrm{CDCl}_{3}\right) \delta 143.9,142.5,141.9,138.3$, 137.0, 136.2, 136.1, 136.0, 135.9, 135.79, 135.76, 135.7, 135.6, 135.3, 135.2, 129.8, 128.6, 126.8, $124.9,124.7,124.4,124.3,124.23,124.21,124.17,124.12,97.8,68.8,62.1,30.6,26.4,25.6,19.5,17.1$, 0.3, 0.20, -4.8; IR (KBr): 3059, 2951, 2926, 2855, 1427, 1078, 986, 833, 804, 791, 773, $473 \mathrm{~cm}^{-1}$; Anal. Calcd for $\mathrm{C}_{40} \mathrm{H}_{46} \mathrm{O}_{2} \mathrm{~S}_{5} \mathrm{Si}_{2}$ : C, 61.97; H, 5.98. Found: C, 61.85; H, 6.08.

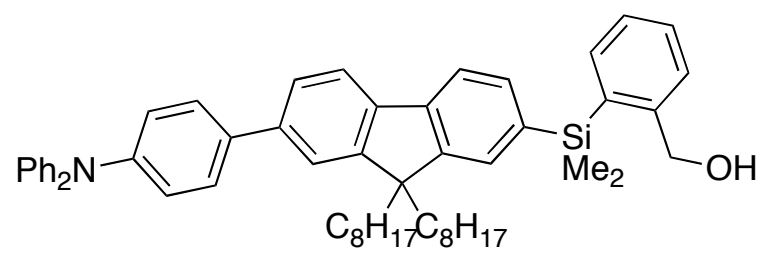

Deprotection of 3ad. To a solution of 3ad $(0.88 \mathrm{~g}, 1.0$ $\mathrm{mmol})$ in $\mathrm{MeOH}(3 \mathrm{~mL})$ and $\mathrm{CH}_{2} \mathrm{Cl}_{2}(3 \mathrm{~mL})$ was added $p$ - $\mathrm{TsOH} \bullet \mathrm{H}_{2} \mathrm{O}(3.8 \mathrm{mg}, 20 \mu \mathrm{mol})$, and the resulting mixture was stirred at $\mathrm{rt}$ overnight. Concentration in vacuo followed by flash chromatography on silica gel to afford $\mathrm{OH}$-free $3 \mathrm{ad}(0.74 \mathrm{~g}, 93 \%)$ as a viscous oil, $\mathrm{R}_{\mathrm{f}}$ 0.27 (hexane-ethyl acetate = 3:1). ${ }^{1} \mathrm{H}$ NMR $\left(400 \mathrm{MHz}, \mathrm{CDCl}_{3}\right) \delta 7.71(\mathrm{~d}, J=7.9 \mathrm{~Hz}, 1 \mathrm{H}), 7.67(\mathrm{~d}, J=$ $7.3 \mathrm{~Hz}, 1 \mathrm{H}), 7.59-7.38(\mathrm{~m}, 9 \mathrm{H}), 7.32-7.21(\mathrm{~m}, 5 \mathrm{H}), 7.18-7.09(\mathrm{~m}, 6 \mathrm{H}), 7.02(\mathrm{t}, J=7.2 \mathrm{~Hz}, 2 \mathrm{H}), 4.54$ $(\mathrm{d}, J=5.9 \mathrm{~Hz}, 2 \mathrm{H}), 2.01-1.98(\mathrm{~m}, 4 \mathrm{H}), 1.30-0.94(\mathrm{~m}, 21 \mathrm{H}), 0.81(\mathrm{t}, J=7.0 \mathrm{~Hz}, 6 \mathrm{H}), 0.74-0.54(\mathrm{~m}$, $10 \mathrm{H}) ;{ }^{13} \mathrm{C}$ NMR $\left(101 \mathrm{MHz}, \mathrm{CDCl}_{3}\right) \delta 151.4,150.2,147.5,146.9,146.5,141.8,139.6,139.5,136.9$, 136.4, 135.5, 135.4, 132.4, 129.8, 129.1, 128.2, 128.0, 127.6, 126.8, 125.3, 124.2, 123.9, 122.8, 120.8, 120.0, 119.2, 65.4, 55.2, 40.3, 31.9, 30.1, 29.31, 29.28, 23.9, 22.7, 14.2, -0.7; IR (neat): 3450, 2953, 2926, 2853, 1591, 1514, 1493, 1464, 1331, 1315, 1279, 837, 816, 752, 696, $502 \mathrm{~cm}^{-1}$; Anal. Calcd for $\mathrm{C}_{56} \mathrm{H}_{67} \mathrm{NOSi}$ : C, 84.26; H, 8.46. Found: C, 84.24; H, 8.23. 


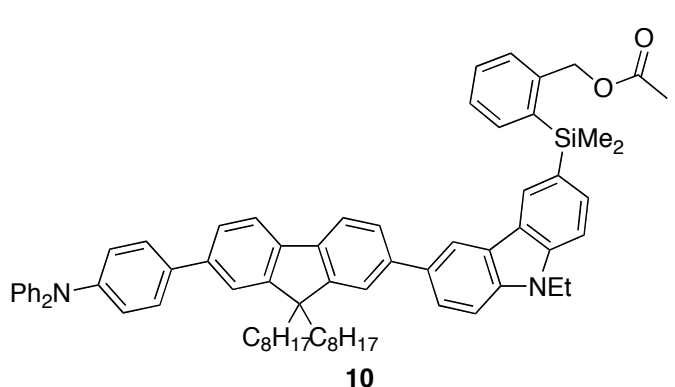

Preparation of 10. To a mixture of deproteced 3ad $(8.0 \mathrm{~g}$, $10 \mathrm{mmol}), \mathrm{K}_{2} \mathrm{CO}_{3}(3.5 \mathrm{~g}, 25 \mathrm{mmol}),\left[\left(\eta^{3}-\mathrm{C}_{3} \mathrm{H}_{5}\right) \mathrm{PdCl}\right]_{2}(18 \mathrm{mg}$, $50 \mu \mathrm{mol}$, measured in a glove box), RuPhos (98 mg, 0.21 $\mathrm{mmol})$, and $\mathrm{CuI}(57 \mathrm{mg}, 0.30 \mathrm{mmol})$ in $\mathrm{DMF}(8 \mathrm{~mL})$ and THF $(22 \mathrm{~mL})$ in a Schlenk tube was added 2'c $(4.8 \mathrm{~g}, 10$ $\mathrm{mmol}$ ), and the resulting mixture was stirred at $75^{\circ} \mathrm{C}$ for 17 $\mathrm{h}$, filtered through a Florisil pad, diluted with $\mathrm{Et}_{2} \mathrm{O}$, washed with water and brine, and then dried over anhydrous $\mathrm{MgSO}_{4}$. Concentration in vacuo followed by flash chromatography on silica gel gave $10(9.0 \mathrm{~g}, 87 \%)$ as a white solid, $\mathrm{mp} 70.8-73.6^{\circ} \mathrm{C}, \mathrm{R}_{\mathrm{f}} 0.37$ (hexane-ethyl acetate $\left.=5: 1\right)$. ${ }^{1} \mathrm{H}$ NMR $\left(400 \mathrm{MHz}, \mathrm{CDCl}_{3}\right) \delta 8.35(\mathrm{~s}, 1 \mathrm{H}), 8.30(\mathrm{~s}, 1 \mathrm{H}), 7.81-7.72(\mathrm{~m}, 3 \mathrm{H}), 7.71-7.62(\mathrm{~m}, 3 \mathrm{H})$, 7.61-7.51 (m, 5H), 7.50-7.32 (m, 5H), 7.31-7.22 (m, 4H), 7.20-7.11 (m, 6H), $7.03(\mathrm{t}, J=7.3 \mathrm{~Hz}, 2 \mathrm{H})$, $5.03(\mathrm{~s}, 2 \mathrm{H}), 4.42(\mathrm{q}, J=7.1 \mathrm{~Hz}, 2 \mathrm{H}), 2.13-1.99(\mathrm{~m}, 4 \mathrm{H}), 1.86(\mathrm{~s}, 3 \mathrm{H}), 1.47(\mathrm{t}, J=7.1 \mathrm{~Hz}, 3 \mathrm{H})$, $1.23-0.98(\mathrm{~m}, 20 \mathrm{H}), 0.84-0.68(\mathrm{~m}, 16 \mathrm{H}) ;{ }^{13} \mathrm{C} \mathrm{NMR}\left(101 \mathrm{MHz}, \mathrm{CDCl}_{3}\right) \delta 170.4,151.5,151.4,147.5$, $146.8,141.2$, 141.0, 139.8, 139.2, 139.1, 139.0, 138.0, 135.8, 135.6, 133.0, 131.2, 129.6, 129.4, 129.1, $127.6,127.5,126.7,126.4,126.0,125.34,125.31,124.2,123.9,123.2,123.0,122.7,121.5,120.8$, $119.74,119.71,118.9,108.5,108.4,66.7,55.3,40.6,37.8,31.9,30.2,29.35,29.32,24.0,22.7,21.0$, 14.2, 14.1, -0.4; IR (KBr): 3452, 2926, 2852, 1738, 1593, 1493, 1464, 1275, 1232, 818, 806, 754, 696 $\mathrm{cm}^{-1}$; Anal. Calcd for $\mathrm{C}_{72} \mathrm{H}_{80} \mathrm{~N}_{2} \mathrm{O}_{2} \mathrm{Si}$ : C, 83.67; H, 7.80. Found: C, 83.92; H, 7.91.

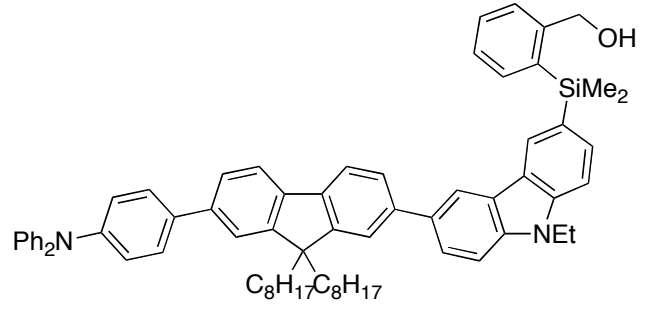
anhydrous $\mathrm{MgSO}_{4}$. Concentration in vacuo followed by flash chromatography on silica gel afforded $\mathrm{OH}$-free $10(0.82 \mathrm{~g}, 95 \%)$ as a white solid, $\mathrm{mp} 81.9-83.5^{\circ} \mathrm{C}, \mathrm{R}_{\mathrm{f}} 0.52$ (hexane-ethyl acetate $\left.=3: 1\right) .{ }^{1} \mathrm{H}$ NMR (400 MHz, $\left.\mathrm{CDCl}_{3}\right) \delta 8.35(\mathrm{~s}, 1 \mathrm{H}), 8.34(\mathrm{~s}, 1 \mathrm{H}), 7.81-7.72(\mathrm{~m}, 3 \mathrm{H}), 7.71-7.62(\mathrm{~m}, 3 \mathrm{H}), 7.61-7.52$ $(\mathrm{m}, 5 \mathrm{H}), 7.51-7.40(\mathrm{~m}, 4 \mathrm{H}), 7.37-7.22(\mathrm{~m}, 5 \mathrm{H}), 7.20-7.11(\mathrm{~m}, 6 \mathrm{H}), 7.03(\mathrm{t}, J=7.2 \mathrm{~Hz}, 2 \mathrm{H}), 4.57(\mathrm{~s}$, $2 \mathrm{H}), 4.40(\mathrm{q}, J=7.1 \mathrm{~Hz}, 2 \mathrm{H}), 2.13-1.97(\mathrm{~m}, 4 \mathrm{H}), 1.47(\mathrm{t}, J=7.1 \mathrm{~Hz}, 3 \mathrm{H}), 1.29-0.98(\mathrm{~m}, 21 \mathrm{H})$, 0.82-0.66 (m, 16H); ${ }^{13} \mathrm{C}$ NMR $\left(101 \mathrm{MHz}, \mathrm{CDCl}_{3}\right) \delta 151.5,151.4,147.5,146.8,146.5,141.0,140.7$, $139.8,139.2$, 139.1, 139.0, 136.7, 135.6, 135.5, 133.0, 131.0, 129.8, 129.1, 128.2, 127.6, 127.2, 126.9, $126.2,126.0,125.4,125.3,124.2,123.9,123.1,122.7,121.5,120.8,119.8,119.7,118.9,108.6,65.4$, $55.3,40.6,37.8,31.9,30.2,29.35,29.32,24.0,22.7,14.2,14.1,-0.4$; IR (KBr): 3450, 2926, 2853, 1593, 1493, 1464, 1275, 1232, 820, 804, 752, $696 \mathrm{~cm}^{-1}$; Anal. Calcd for $\mathrm{C}_{70} \mathrm{H}_{78} \mathrm{~N}_{2} \mathrm{OSi}: \mathrm{C}, 84.80 ; \mathrm{H}$, 7.93. Found: C, 84.79; H, 7.84 .

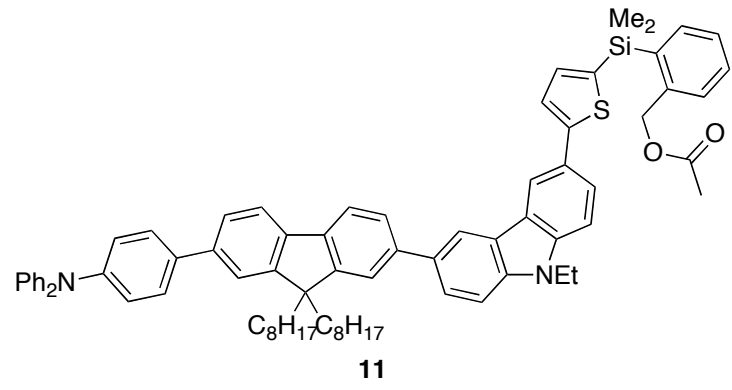

Synthesis of 11. To a mixture of deacetylated $\mathbf{1 0}(0.99 \mathrm{~g}$, $1.0 \mathrm{mmol}), \mathrm{K}_{2} \mathrm{CO}_{3}(0.99 \mathrm{~g}, 1.0 \mathrm{mmol}),\left[\left(\eta^{3}-\mathrm{C}_{3} \mathrm{H}_{5}\right) \mathrm{PdCl}\right]_{2}$ (9.1 mg, $25 \mu \mathrm{mol}$, measured in a glove box), RuPhos (49 $\mathrm{mg}, 0.10 \mathrm{mmol})$, and $\mathrm{CuI}(9.5 \mathrm{mg}, 50 \mu \mathrm{mol})$ in $\mathrm{DMF}(0.8$ $\mathrm{mL})$ and THF (2.2 $\mathrm{mL})$ in a Schlenk tube was added 2'b $(0.37 \mathrm{~g}, 1.0 \mathrm{mmol})$, and the resulting mixture was stirred at $75{ }^{\circ} \mathrm{C}$ for $24 \mathrm{~h}$. The mixture was filtered through a Florisil pad, diluted with $\mathrm{Et}_{2} \mathrm{O}$, washed with water and brine, and dried over anhydrous $\mathrm{MgSO}_{4}$. After concentration in vacuo, the residue was purified by flash 
chromatography on silica gel to give $11(0.87 \mathrm{~g}, 78 \%)$ as a yellow solid, $\mathrm{mp} 71.8-73.6{ }^{\circ} \mathrm{C}, \mathrm{R}_{\mathrm{f}} 0.38$ (hexane-ethyl acetate $=5: 1) .{ }^{1} \mathrm{H}$ NMR $\left(400 \mathrm{MHz} \mathrm{CDCl}_{3}\right) \delta 8.40(\mathrm{~d}, J=1.1 \mathrm{~Hz}, 1 \mathrm{H}), 8.39(\mathrm{~s}, 1 \mathrm{H})$, 7.83-7.52 (m, 11H), 7.51-7.32 (m, 6H), 7.31-7.21 (m, 5H), 7.20-7.11 (m, 6H), 7.03 (t, J = 7.3 Hz, 2H), $5.14(\mathrm{~s}, 2 \mathrm{H}), 4.40(\mathrm{q}, J=7.1 \mathrm{~Hz}, 2 \mathrm{H}), 2.15-1.96(\mathrm{~m}, 7 \mathrm{H}), 1.48(\mathrm{t}, J=7.2 \mathrm{~Hz}, 3 \mathrm{H}), 1.22-0.99(\mathrm{~m}, 24 \mathrm{H})$, $0.77(\mathrm{t}, J=7.0 \mathrm{~Hz}, 6 \mathrm{H}), 0.72(\mathrm{~s}, 6 \mathrm{H}) ;{ }^{13} \mathrm{C} \mathrm{NMR}\left(101 \mathrm{MHz}, \mathrm{CDCl}_{3}\right) \delta 170.5,151.8,151.5,151.4,147.5$, $146.8,141.1,140.6,139.9$, 139.7, 139.6, 139.2, 139.0, 136.9, 136.4, 136.1, 135.6, 135.5, 133.0, 129.9, $129.5,129.1,127.6,126.0,125.5,125.3,124.4,124.2,123.9,123.40,123.36,122.7,121.4,120.8$, 119.8, 119.7, 118.9, 118.0, 108.8, 66.6, 55.3, 40.6, 37.9, 31.9, 30.2, 29.34, 29.32, 24.0, 22.7, 21.1, 14.2, 14.1, 0.3; IR (KBr): 3466, 2926, 2855, 1736, 1593, 1493, 1483, 1466, 1275, 1252, 1231, 804, 752,696 $\mathrm{cm}^{-1}$; Anal. Calcd for: C, 81.82; H, 7.41. Found: C, 81.57; H, 7.51.

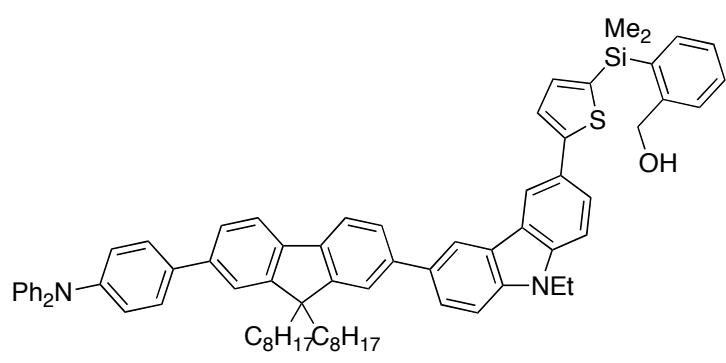

Deprotection of 11. To a solution of $11(0.82 \mathrm{~g}, 0.74$ mmol) in $\mathrm{CH}_{2} \mathrm{Cl}_{2}(2 \mathrm{~mL})$ was added a $1.5 \mathrm{M}$ solution of DIBAL-H $(0.81 \mathrm{mmol})$ in toluene at $-78{ }^{\circ} \mathrm{C}$, and the resulting mixture was stirred at the same temperature for 2 h. The reaction was quenched with a saturated $\mathrm{NH}_{4} \mathrm{Cl}$ aqueous solution at $-78{ }^{\circ} \mathrm{C}$, diluted with $\mathrm{Et}_{2} \mathrm{O}$, and slowly warmed to $\mathrm{rt}$ The aqueous layer was extracted with diethyl ether, and the combined organic layers were dried over anhydrous $\mathrm{MgSO}_{4}$. After concentration in vacuo, the residue was purified by flash chromatography on silica gel to afford deacetylated $11(0.68 \mathrm{~g}, 85 \%)$ as a yellow solid, mp 86.7-88.0 ${ }^{\circ} \mathrm{C}, \mathrm{R}_{\mathrm{f}} 0.50$ (hexane-ethyl acetate = 3:1). ${ }^{1} \mathrm{H}$ NMR $\left(400 \mathrm{MHz}, \mathrm{CDCl}_{3}\right) \delta 8.40(\mathrm{~s}, 1 \mathrm{H}), 8.39(\mathrm{~s}$, $1 \mathrm{H}), 7.83-7.37(\mathrm{~m}, 15 \mathrm{H}), 7.36-7.21(\mathrm{~m}, 7 \mathrm{H}), 7.20-7.10(\mathrm{~m}, 6 \mathrm{H}), 7.03(\mathrm{td}, J=7.3,1.0 \mathrm{~Hz}, 2 \mathrm{H}), 4.72(\mathrm{~d}$, $J=5.9 \mathrm{~Hz}, 2 \mathrm{H}), 4.41(\mathrm{q}, J=7.1 \mathrm{~Hz}, 2 \mathrm{H}), 2.14-1.98(\mathrm{~m}, 4 \mathrm{H}), 1.48(\mathrm{t}, J=7.2 \mathrm{~Hz}, 3 \mathrm{H}), 1.22-0.99(\mathrm{~m}$, $25 \mathrm{H}), 0.77(\mathrm{t}, J=6.8 \mathrm{~Hz}, 6 \mathrm{H}), 0.72(\mathrm{~s}, 6 \mathrm{H}) ;{ }^{13} \mathrm{C} \mathrm{NMR}\left(101 \mathrm{MHz}, \mathrm{CDCl}_{3}\right) \delta 151.5,151.4,149.5,147.5$, $146.8,145.6,140.6,139.8,139.72$, 139.65, 139.2, 139.0, 135.6, 134.9. 133.0, 130.8, 129.4, 129.1, $127.9,127.6,126.7,126.0,125.7,125.6,125.4,124.4,124.2,123.9,123.5,123.4,123.3,122.7,121.9$, $121.51,121.48,120.8,119.8,119.7,118.9,118.0,108.80,108.77,71.5,55.3,40.6,37.9,31.9,30.2$, 29.4, 29.3, 24.0, 22.7, 14.2, 14.0, 0.7; IR (KBr): 3452, 2926, 2853, 1593, 1493, 1466, 1275, 804, 752, $696 \mathrm{~cm}^{-1}$; Anal. Calcd for $\mathrm{C}_{74} \mathrm{H}_{80} \mathrm{~N}_{2} \mathrm{OSSi}$ : C, 82.79; H, 7.51. Found: C, 82.53; H, 7.54.

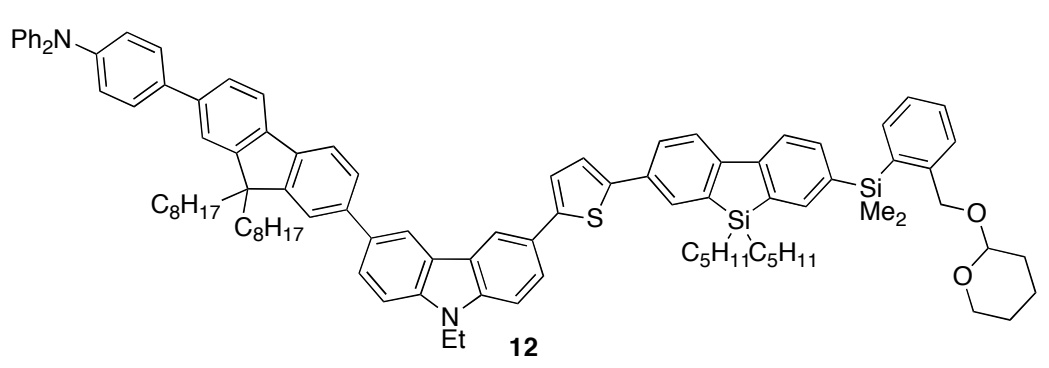

Synthesis of 12. To a mixture of deprotected 11 (107 $\mathrm{mg}, 0.10 \mathrm{mmol}$ ), $\mathrm{K}_{2} \mathrm{CO}_{3} \quad(35 \quad \mathrm{mg}, \quad 0.25 \quad \mathrm{mmol})$, (dppf) $\mathrm{PdCl}_{2} \bullet \mathrm{CH}_{2} \mathrm{Cl}_{2}(4.1 \mathrm{mg}, 5.0 \mu \mathrm{mol})$, and $\mathrm{CuI}(1.0 \mathrm{mg}, 5.0 \mu \mathrm{mol})$ in $\mathrm{DMF}$ $(80 \mu \mathrm{L})$ and $\operatorname{THF}(0.22 \mathrm{~mL})$ in a Schlenk tube was added 2e (65 $\mathrm{mg}$, $0.10 \mathrm{mmol}$ ), and the resulting mixture was stirred at $75^{\circ} \mathrm{C}$ for $24 \mathrm{~h}$. The mixture was filtered through a Florisil pad, diluted with $\mathrm{Et}_{2} \mathrm{O}$, washed with water and brine, and dried over anhydrous $\mathrm{MgSO}_{4}$. Concentration in vacuo followed by flash chromatography on silica gel gave $12(0.118 \mathrm{~g}, 80 \%)$ as a yellow solid, mp $91.7-94.0{ }^{\circ} \mathrm{C}, \mathrm{R}_{\mathrm{f}} 0.35$ (hexane-ethyl acetate $=10: 1) .{ }^{1} \mathrm{H}$ NMR $\left(400 \mathrm{MHz} \mathrm{CDCl}_{3}\right) \delta 8.45(\mathrm{~s}, 1 \mathrm{H}), 8.43(\mathrm{~s}, 1 \mathrm{H}), 7.91-7.65(\mathrm{~m}$, $10 \mathrm{H}), 7.62-7.33(\mathrm{~m}, 12 \mathrm{H}), 7.32-7.11(\mathrm{~m}, 12 \mathrm{H}), 7.03(\mathrm{t}, J=7.2 \mathrm{~Hz}, 2 \mathrm{H}), 4.70(\mathrm{~d}, J=11.9 \mathrm{~Hz}, 2 \mathrm{H})$, 4.51-4.38 (m, 4H), 3.82-3.72 (m, 1H), 3.46-3.36 (m, 1H), 2.18-1.99 (m, 4H), 1.85-1.72 (m, 1H), $1.68-0.91(\mathrm{~m}, 47 \mathrm{H}), 0.89-0.70(\mathrm{~m}, 12 \mathrm{H}), 0.63(\mathrm{~s}, 6 \mathrm{H}) ;{ }^{13} \mathrm{C} \mathrm{NMR}\left(101 \mathrm{MHz}, \mathrm{CDCl}_{3}\right) \delta 151.5,151.4$, $148.5,147.5,147.2,146.8,144.7,144.0,142.5,140.6,139.8,139.72,139.68,139.2,139.1,138.9$, $138.7,137.2$, 136.9, 136.3, 136.0, 135.6, 135.5, 133.3, 133.0, 129.9, 129.4, 129.1, 128.4, 127.6, 127.1, 
ja074728s_SI_0824.pdf

$126.7,126.0,125.7,125.6,125.4,124.2,124.0,123.9,123.7,123.5,123.3,122.8,122.7,121.4,121.2$, $120.8,120.0,119.8,119.7,118.9,117.6,108.9,108.8,97.7,68.8,62.0,55.3,40.6,37.9,35.6,31.9$, $30.6,30.2,29.8,29.3,25.5,24.0,23.7,22.7,22.3,19.4,14.2,14.13,14.07,12.4,-0.7,-0.8$; IR (KBr): 2924, 2855, 1591, 1493, 1462, 1292, 1277, 1232, 837, 820, 802, 752, $694 \mathrm{~cm}^{-1}$; Anal. Calcd for $\mathrm{C}_{101} \mathrm{H}_{116} \mathrm{~N}_{2} \mathrm{O}_{2} \mathrm{SSi}_{2}$ : C, 82.06; H, 7.91. Found: C, 81.90; H, 7.89.

\section{Reference}

1. Pangborn, A. B.; Giardello, M. A.; Grubbs, R. H.; Rosen, R. K.; Timmers, F. J. Organometallics 1996, 15, 1518-1520.

2. Milne, J. E.; Buchwald, S. L. J. Am. Chem. Soc. 2004, 126, 13028.

3. (a) Nakao, Y.; Imanaka, H.; Sahoo, A. K.; Yada, A.; Hiyama, T. J. Am. Chem. Soc. 2005, 127, 6952. (b) Nakao, Y.; Sahoo, A. K.; Yada, A.; Chen, J.; Hiyama, T. Science and Technology of Advanced Materials 2006, 7, 536. (c) Nakao, Y.; Imanaka, H.; Chen, J.; Yada, A.; Hiyama, T. J. Organomet. Chem. 2007, 692, 585. (d) Nakao, Y.; Ebata, S.; Chen, J.; Imanaka, H.; Hiyama, T. Chem. Lett. 2007, 36, 606. (e) Nakao, Y.; Imanaka, H.; Chen, J.; Hiyama, T.; Ichikawa, Y.; Duan, W.; Shintani R.; Hayashi, T. J. Am. Chem. Soc. 2007, 129, 9137.

4. Li, Z. H.; Wong, M. S. Org. Lett. 2006, 8, 1499.

5. $\quad$ Wong, K.; Chen Y.; Lin, Y.; Su, H.; Wu, C. Org. Lett. 2005, 7, 5361.

6. Chan, K. L.; McKiernan, M. J.; Towns, C. R.; Holmes, A. B. J. Am. Chem. Soc. 2005, 127, 6952. 


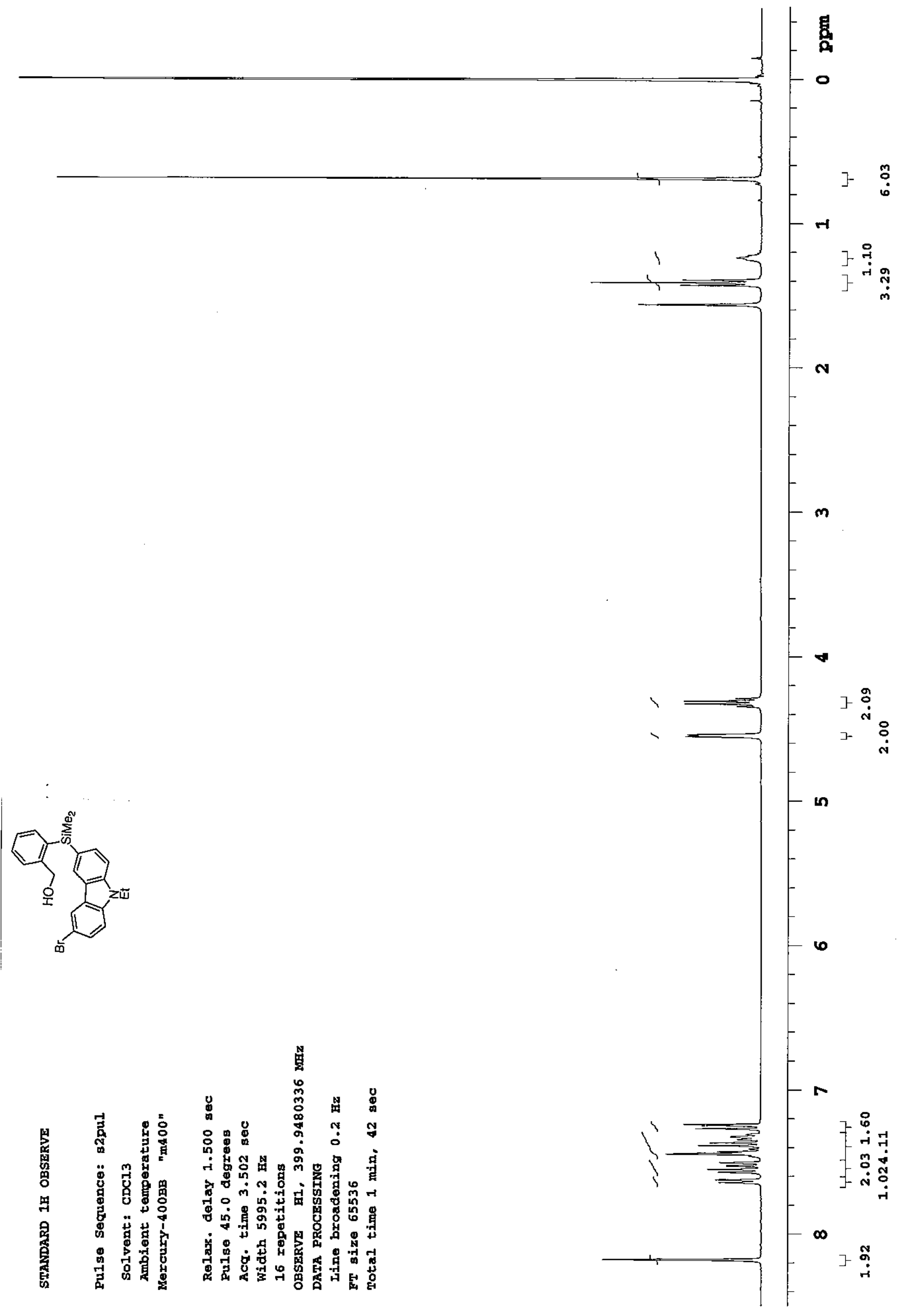


ja074728s_SI_0824.pdf

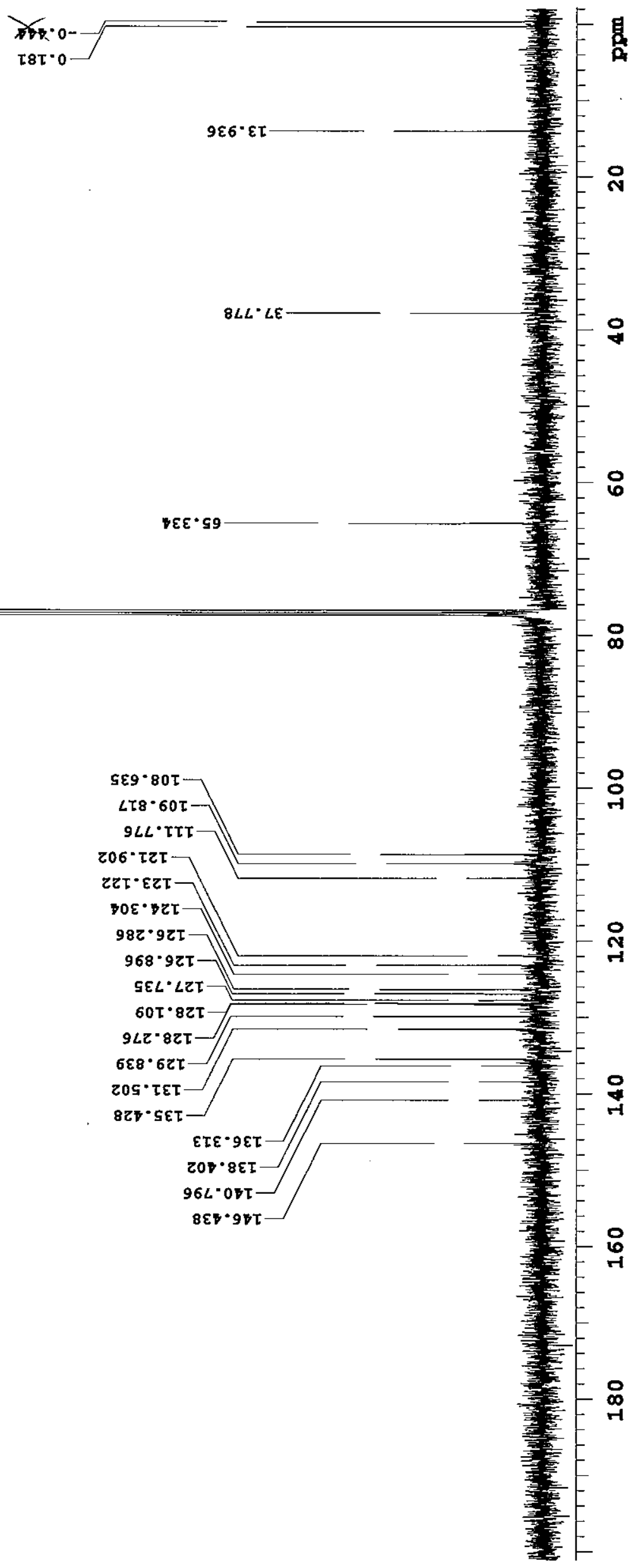




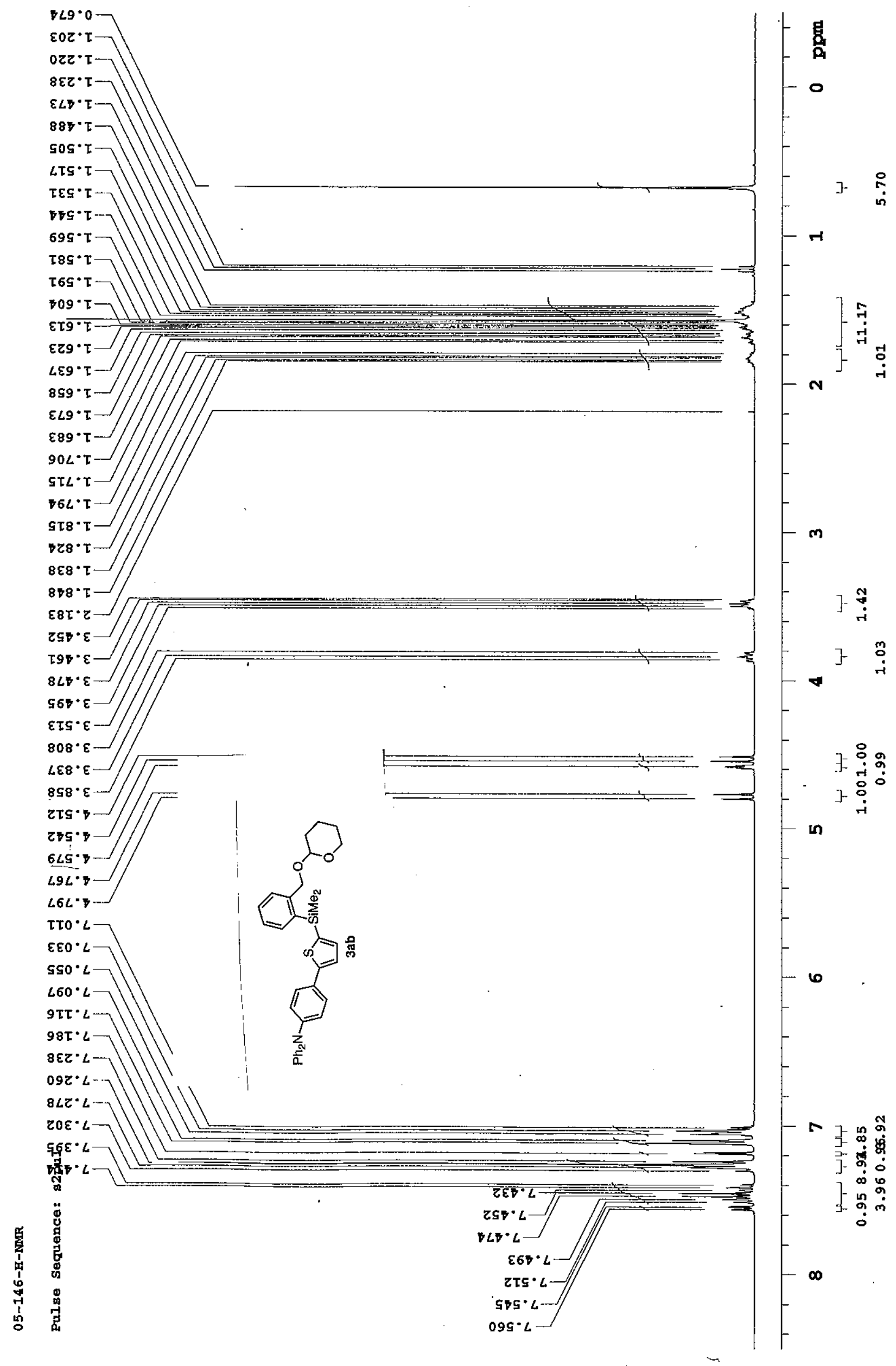



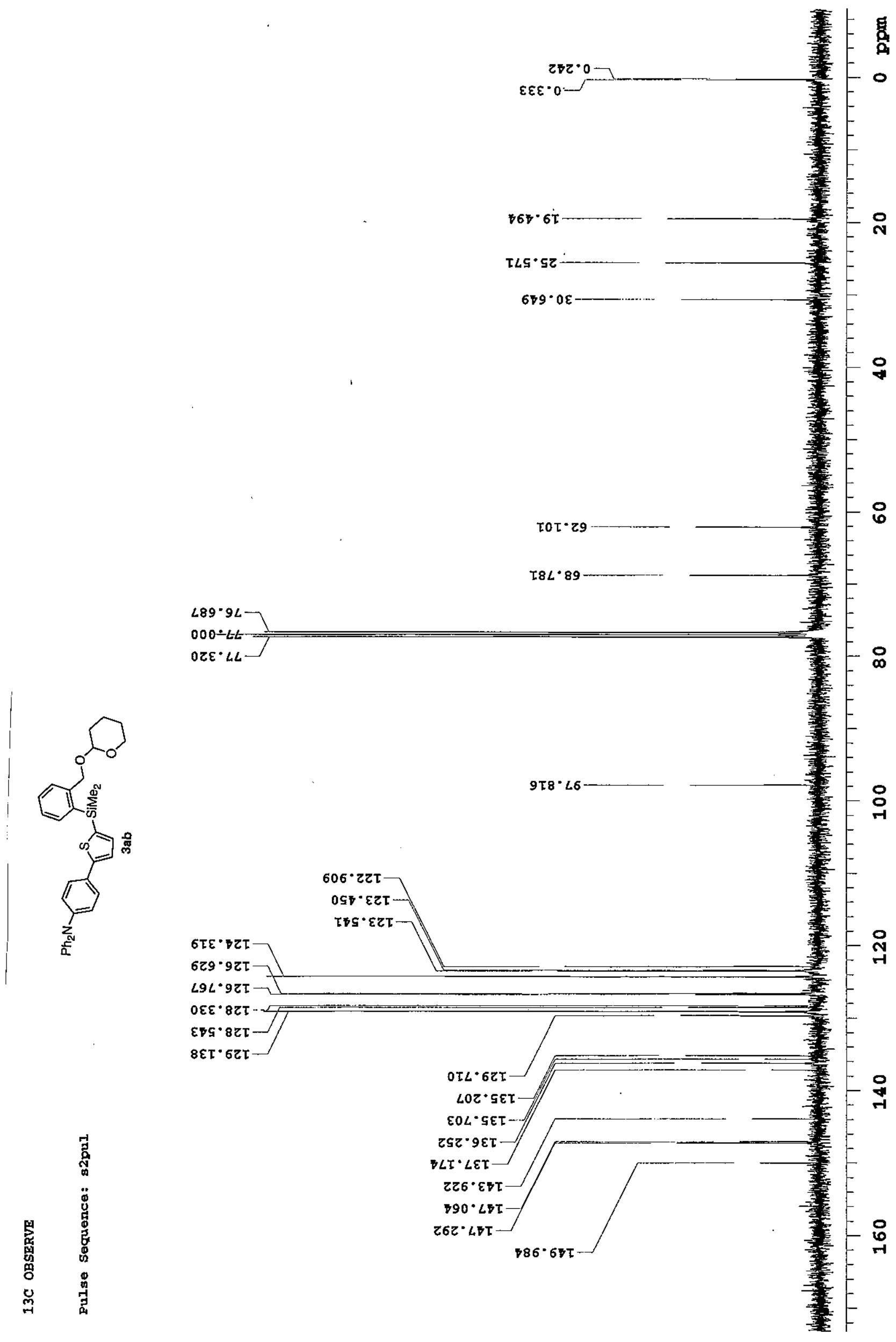


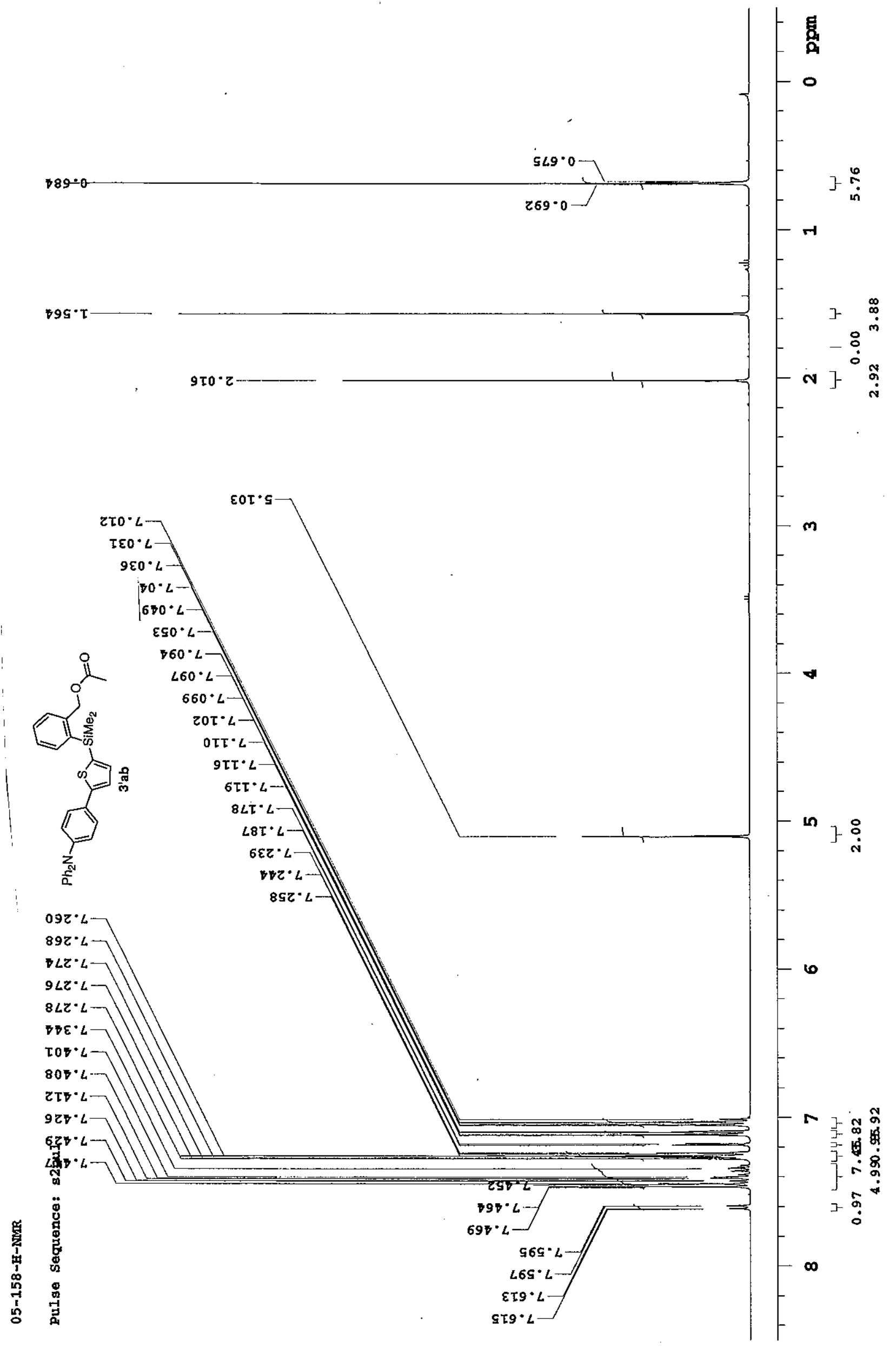


ja074728s_SI_0824.pdf

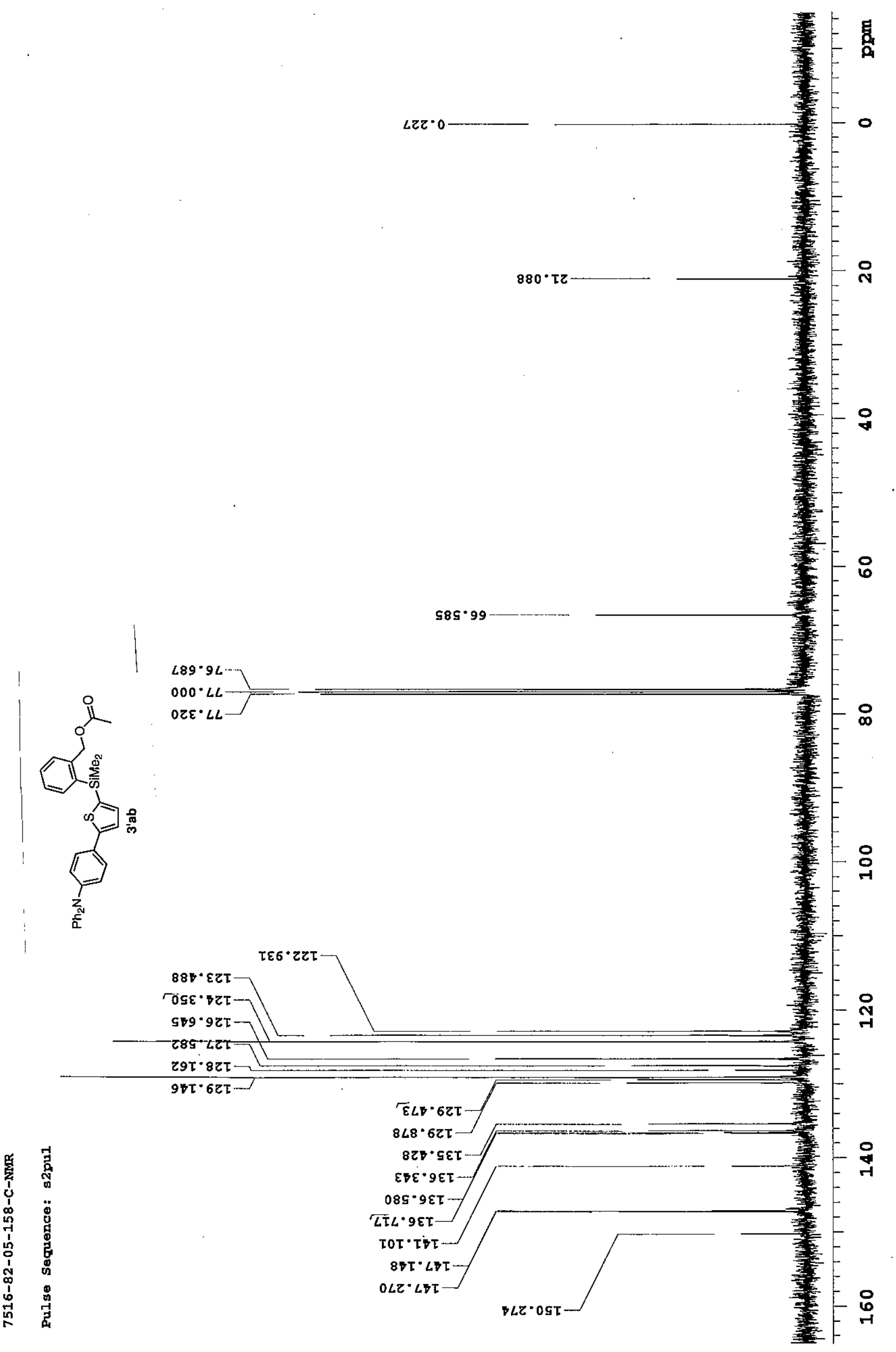




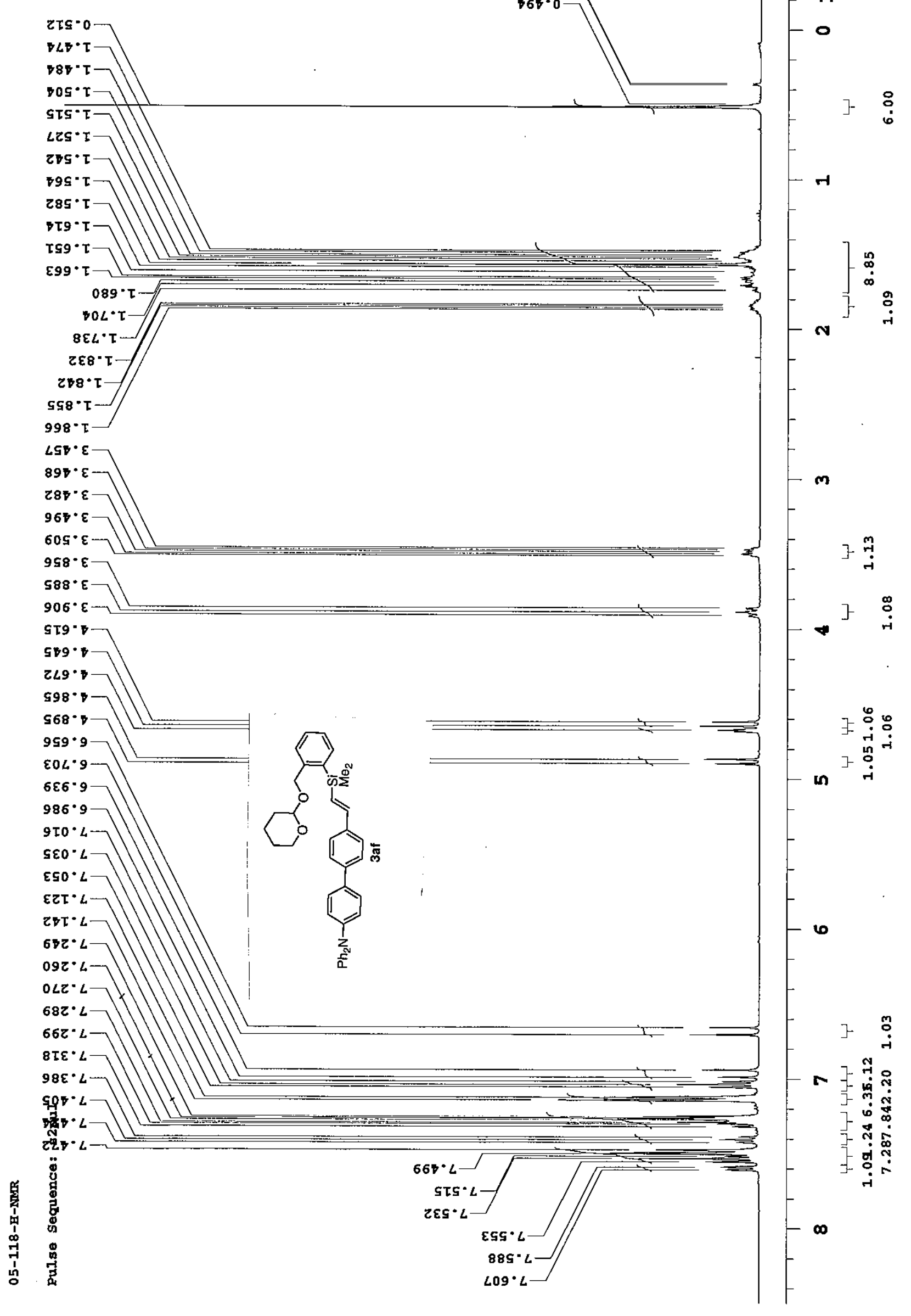




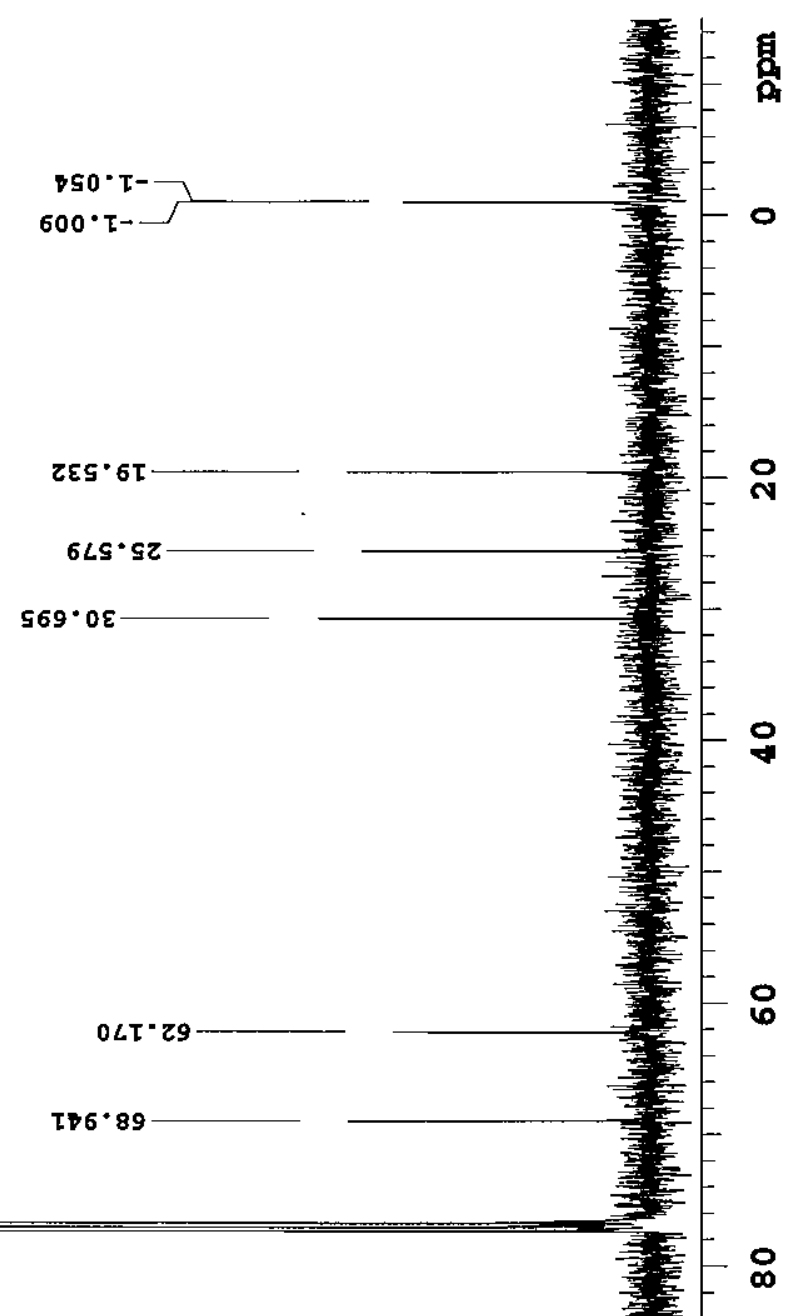

$\angle 89 \cdot 9 L$
$058 \cdot 9 L$
$000 \cdot \angle L$

ozE: LL
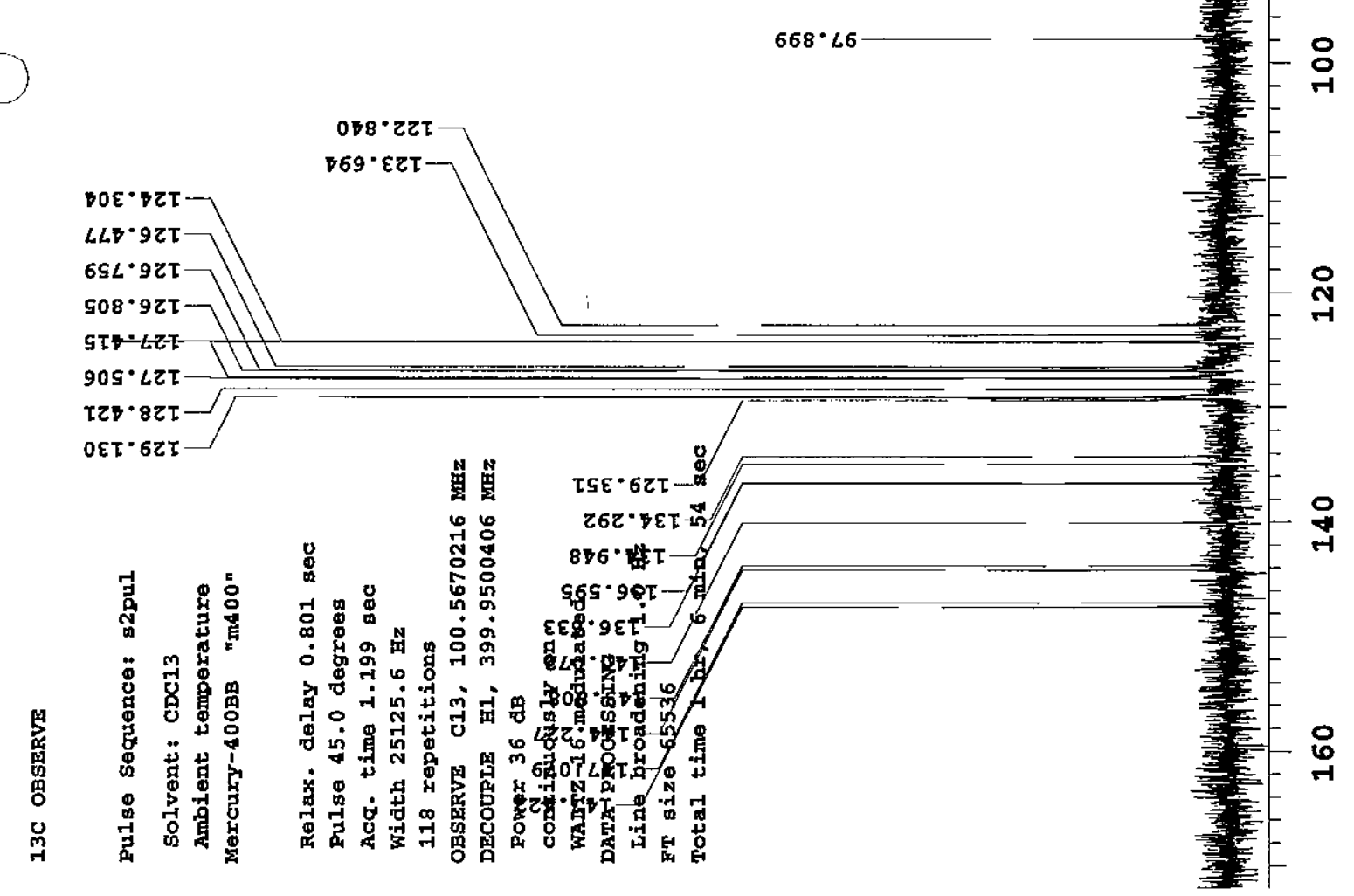


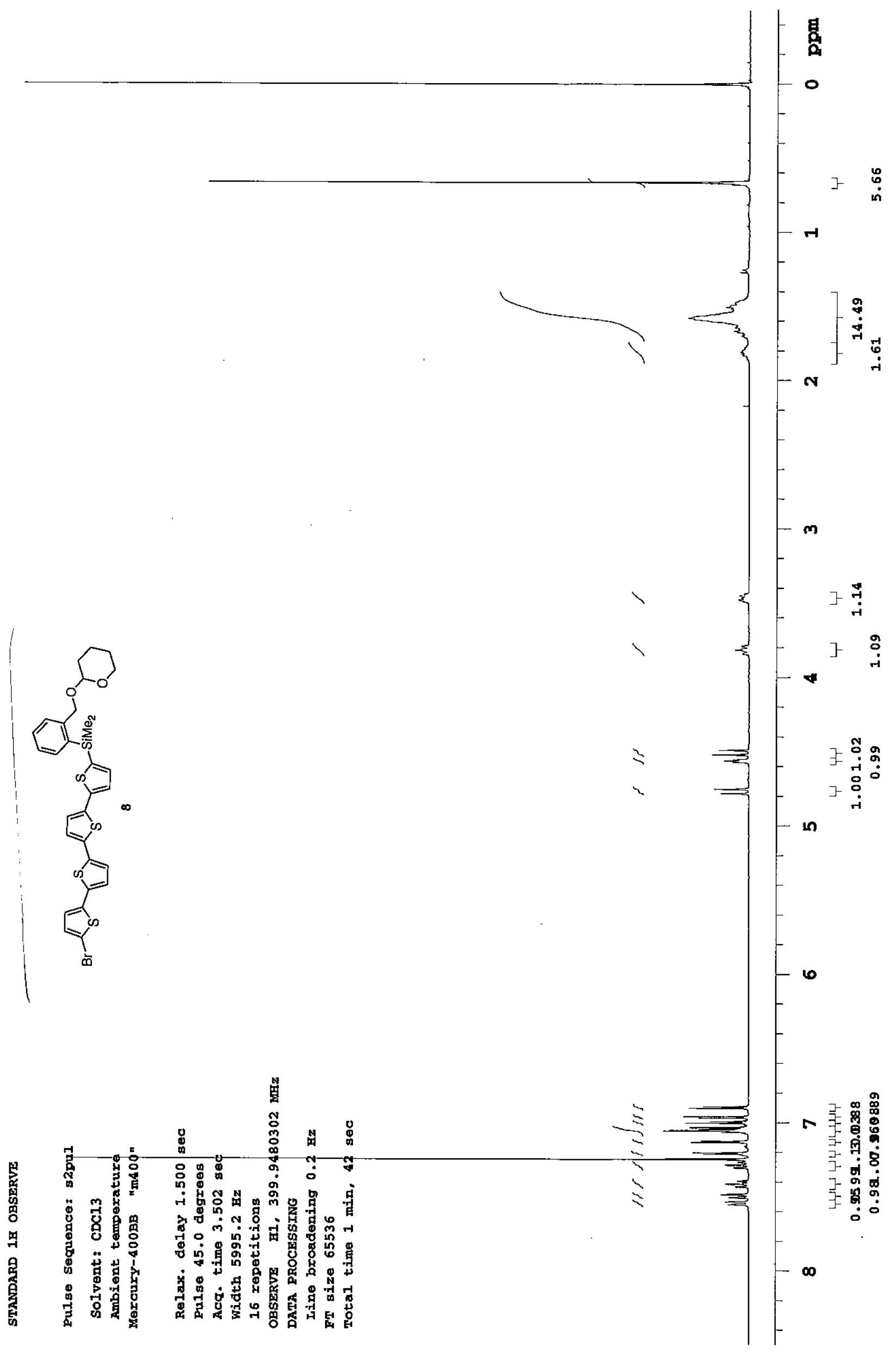



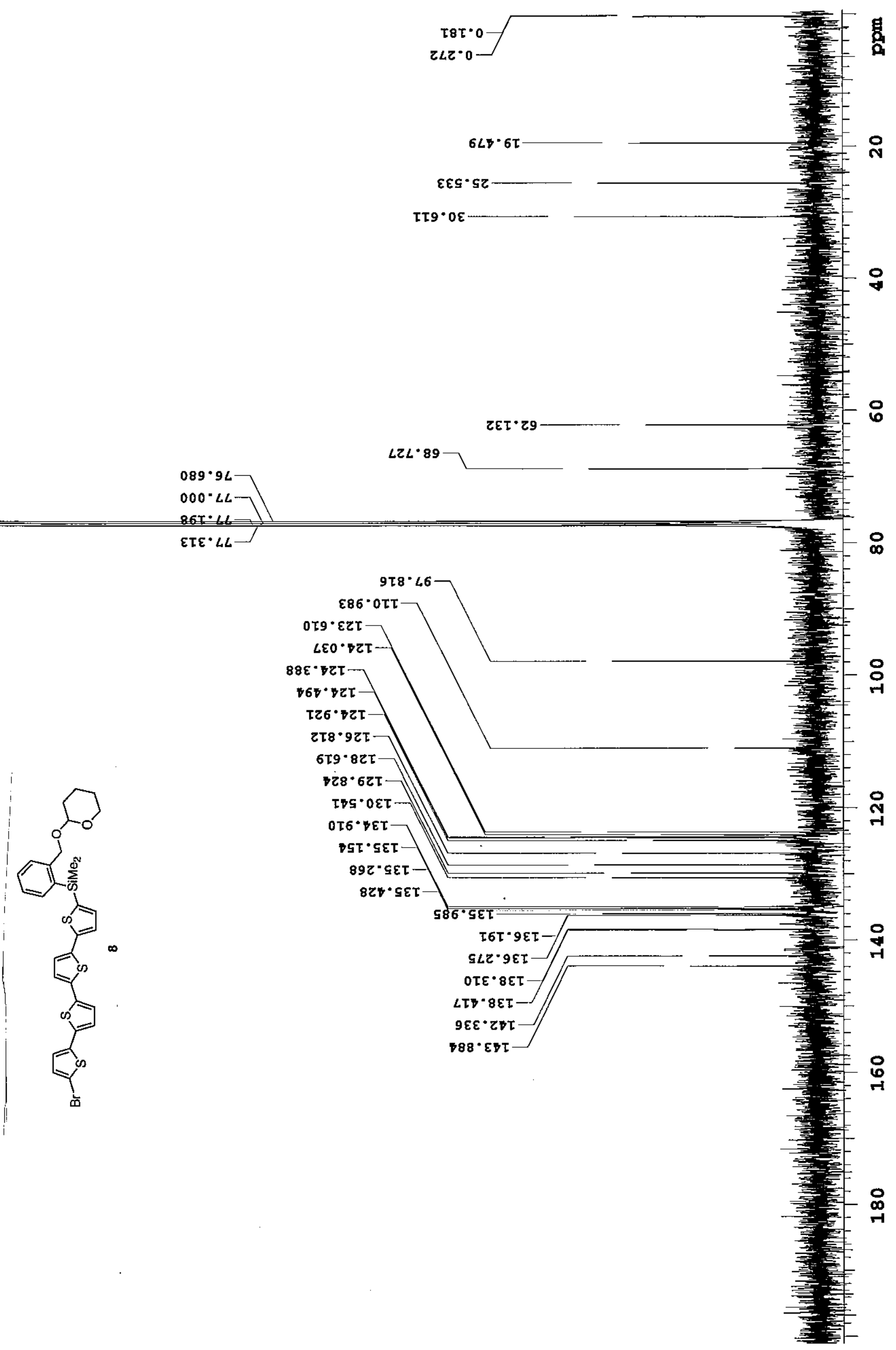